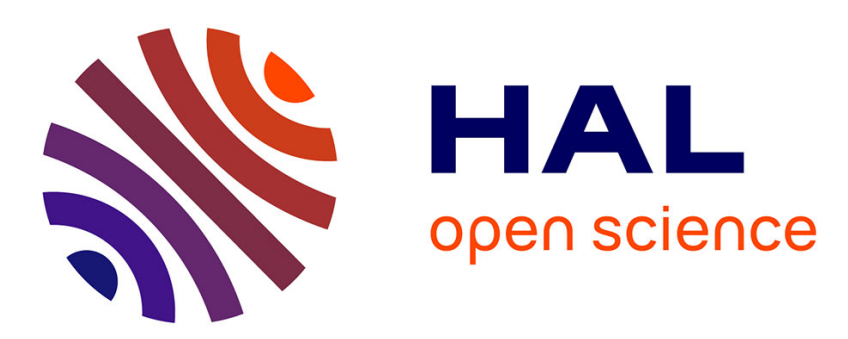

\title{
A new algorithm for solving the multi-indentation problem of rigid bodies of arbitrary shapes on a viscoelastic half-space
}

Ivan Kozhevnikov, Denis Duhamel, Yin Hai-Ping, Zhi Qiang Feng

\section{- To cite this version:}

Ivan Kozhevnikov, Denis Duhamel, Yin Hai-Ping, Zhi Qiang Feng. A new algorithm for solving the multi-indentation problem of rigid bodies of arbitrary shapes on a viscoelastic half-space. International Journal of Mechanical Sciences, 2010, 52 (3), pp.399-409. 10.1016/j.ijmecsci.2009.10.015 . hal-00517335

\section{HAL Id: hal-00517335 \\ https://hal.science/hal-00517335}

Submitted on 14 Sep 2010

HAL is a multi-disciplinary open access archive for the deposit and dissemination of scientific research documents, whether they are published or not. The documents may come from teaching and research institutions in France or abroad, or from public or private research centers.
L'archive ouverte pluridisciplinaire HAL, est destinée au dépôt et à la diffusion de documents scientifiques de niveau recherche, publiés ou non, émanant des établissements d'enseignement et de recherche français ou étrangers, des laboratoires publics ou privés. 


\title{
A new algorithm for solving the
} multi-indentation problem of rigid bodies of arbitrary shapes on a viscoelastic half-space

\author{
I.F. Kozhevnikov ${ }^{\text {a,* }}$, D. Duhamel ${ }^{\text {b }}$, H.P. Yin ${ }^{\text {b }}$, Z.-Q. Feng ${ }^{c}$ \\ ${ }^{a}$ Dorodnicyn Computing Centre of the Russian Academy of Sciences, Vavilov st. \\ 40, 119333, Moscow, Russia \\ ${ }^{\mathrm{b}}$ Université Paris-Est, UR Navier, ENPC, 6-8 Av. Blaise Pascal, 77455 Marne la \\ Vallée, Cedex 2, France \\ ${ }^{\mathrm{c}}$ Université d'Evry, 40 rue du Pelvoux, 91020 Evry, France
}

\begin{abstract}
In this paper the contact problem between rigid indenters of arbitrary shapes and a viscoelastic half-space is considered. Under the action of a normal force the penetration of the indenters changes and a few contact areas appeared. We wish to find the relations which link the pressure distribution, the resultant forces on the indenters and the penetration on the assumption that the surfaces are frictionless. For indenters of arbitrary shapes the problem may be solved numerically by using the Matrix Inversion Method, extended to viscoelastic cases [1]. But when the problem involves a large number of points the Matrix Inversion Method can become very time-consuming. Here the problem is solved using an alternative scheme, called the Two-scale Iterative Method. In this method the Local Matrix Inversion Method is used at the micro-scale for each contact area to compute the pressure distribution taking into account interacting effect (the forces on the other contact areas which
\end{abstract}


can be calculated at the macro-scale) between indenters. Two algorithms were proposed. The first algorithm takes into account the distribution of forces on the other contact areas and the second is the approximation of the first algorithm and takes into account the resultant forces on the other contact areas. The method was implemented for a simple configuration of seven spherical indenters, seven spherical-ended cylindrical indenters and seven flat-ended cylindrical indenters as well as for a more complex configuration of twelve randomly positioned indenters of arbitrary shapes: spherical-ended cylindrical, flat-ended cylindrical, conical and cylindrical indenters (finite cylindrical shape with its curved face). This last case is more difficult as the indenting geometry doesn't have an axisymmetric profile. For all these cases the Two-scale Iterative Method permits to find the pressure distribution and the contact forces versus the penetration. It can be validated by comparing the numerical results to the numerical results obtained with the Matrix Inversion Method.

Key words: Contact mechanics, Viscoelastic contact, Viscoelasticity, Indenter, Numerical methods

\section{Introduction}

The contact between a tyre and a road is mainly modeled in the frame of the elastic theory. Generally, the viscoelastic effects have not been taken into account for computing the pressure distribution. But in fact the dynamic modulus of rubber is frequency dependent and the viscoelastic part can be more important than the elastic modulus. So the aim of our work is to propose a new numerical method for problems of multi-indentation on a viscoelastic halfspace with asperities of arbitrary shapes for an increasing and then decreasing

\footnotetext{
* Corresponding author : Tel: (7 499) 1353590 ; Fax: (7 499) 1356159

Email address: kogevnik@ccas.ru (I.F. Kozhevnikov).
} 
vertical loading.

In the past, Boussinesq [2], using the potential theory, investigated the normal contact between a perfectly rigid indenter and a plane elastic half-space. In the case of two elastic bodies with smooth and quadratic contacting surfaces, Hertz $[3,4]$ also gave an analytical solution of the contact problem. Numerous analytical or semi-analytical solutions were then derived from Boussinesq's theory for a rigid indenter of arbitrary shape on an elastic half-space, especially in axisymmetric contact cases by Sneddon [5]. In the paper by Vilke [6] an invariant form for the force function which depends on the geometric properties of an intersection for the undeformed rigid bodies is proposed. This expression is supposed to hold in cases when the classic contact theory hypotheses fail.

The problem of a rigid indenter pressed into contact with a viscoelastic solid was also investigated by many authors. For finding the stresses and deformations in cases where the corresponding solution for an elastic material is known one can use a suggestion by Radok [7], which consists in replacing the elastic constant in the elastic solution by the corresponding integral operator from the viscoelastic stress-strain relations. This approach can be applied to the contact problem if the contact area is increasing and can not be used if the contact area decreases. This complication has been studied by Ting [8] for a rigid axisymmetric indenter. Recently Vandamme and Ulm [9] show that for conical indenters the suggestion of Radok remains valid at the very beginning of the unloading phase as well.

Analytical, numerical and experimental studies have been made in case of spherical, conical and pyramid indenters. Ball (Brinell) and flat (Boussinesq) punch indentation was analysed in the work of Larsson and Carlsson [10], the 
elliptic indenter in the work of Yang [11], pyramid indenters in the works of Giannakopoulos [12], Murakami et al. [13], Larsson et al. [14], Rabinovich and Savin [15]. Cheng et al. [16] analyse the indentation of viscoelastic solids by a spherical-tip indenter.

The contact problem when the number of indenters is large, was also investigated by many authors. Greenwood and Williamson [17] proposed a statistical method for contacts between rough surfaces. Asperities were approached by independent spheres from the statistical properties of the surfaces and the Hertz's theory was used. Such methods don't take into account the interaction between asperities. McCool [18] has made numerical comparisons between the Greenwood-Williamson elastic model and two more general isotropic and anisotropic models. In the work by Carbone and Bottiglione [19] a critical analysis of the principal contact theories (based on the idea of Greenwood and Williamson) of rough surfaces was made. In the paper by Kucharski et al. [20] the elastoplastic asperity-based model for the contact of rough surfaces is presented. This model adopts most of the basic asperity-based assumptions, introducing a more realistic elastoplastic deformation law for the analysis of individual asperity deformations. The paper by Adams and Nosonovsky [21] reviews contact modeling with an emphasis on the contact forces and their relationship to the geometrical, material and mechanical properties of the contacting bodies. The paper by Cesbron et al. [22] deals with the experimental study of dynamical tyre/road contact for noise prediction. The measurements of contact forces were carried out for a slick tyre rolling on six different road surfaces between 30 and $50 \mathrm{~km} / \mathrm{h}$. The contact areas obtained during rolling were smaller than in static conditions, that is mainly explained by the dynamical properties of tyre compounds, like the viscoelastic behaviour of the rubber. 
One can also note the works by Kragelsky and Demkin [23], O'Callaghan and Cameron [24], Bush et al. [25].

For the analysis of the contact between rough surfaces the iterative approach was also used in the literature ( Singh and Paul [26], Webster and Sayles [27], Ju and Zheng [28] and Chang and Gao [29] ). These methods can become time-consuming when the number of unknowns increases. A numerical approach was presented in the paper by Nayak and Johnson [30] for calculating the pressure distribution and the contact area shape between two elastic bodies of arbitrary profile. Nowell and Hills [31] propose another method for solving multi-contact problems for normal pressure which was extended later to shearing forces by Ciavarella et al. [32]. In this approach any individual asperity contact produces a constant normal displacement at the other asperity sites. This hypothesis is correct if the asperities are not close. The paper by Karpenko and Akay [33] describes a computational method to calculate the friction force between two rough surfaces, which shows how friction changes with surface roughness. The analysis of real surface contacts and pressure distributions in sliding wear was also made in the article by Liu et al. [34]. Stanley and Kato [35] used yet another numerical method based on a Fast Fourier Transform (FFT) from spatial domain to wavelength domain of the contacting surfaces. This procedure may be used even for contact problems with a large number of asperities and it is limited for finding the contact solution at large wavelengths due to an aliasing introduced by the FFT.

The Matrix Inversion Method (MIM) (Kalker [36]) is described in the book of Johnson [37]. In the MIM the boundary conditions are satisfied exactly at specified "matching points" (the mid-points of the boundary elements). The MIM is used in the work of Cesbron et al. [38] for the analysis of the elas- 
tic tyre-road contact. In this method, called the Two-scale Iterative Method (TIM), one proceeds successively at two scales. At the macro-scale, the forces on each indenter are computed from an approximate relation between the normal contact force and the relative displacement of the tip of each indenter. The approximate relation takes into account the interacting effect between the indenters. At the micro-scale the Local Matrix Inversion Method (LMIM) is used to compute the pressure distribution on each contact area by an iterative scheme.

In the paper of Kozhevnikov et al. [1] a new algorithm for computing the indentation of a rigid body of arbitrary shape on a viscoelastic half-space was proposed. In this method the MIM was extended to viscoelastic problems. It can be validated by comparing the numerical results to the analytic solutions in cases of a spherical asperity (loading and unloading) and a conical asperity (loading only). Finally, the method was implemented for a finite cylindrical shape with its curved face indenting the surface of the half-space. But when the problem involves a large number of points the MIM can become very time-consuming.

In this paper the problem is solved using the TIM. In this method the LMIM is used at the micro-scale for each contact area to compute the pressure distribution taking into account interacting effects (the forces on the other contact areas which can be calculated at the macro-scale) between indenters.

The paper is structured as follows. The indentation by a rigid indenter of arbitrary shape will be considered first. Then the discretization of the contact problem will be made and two algorithms will be proposed. Next it will be possible to solve the general contact problem by the TIM. The general 
methodology will be applied to the simple configuration of seven spherical indenters, seven spherical-ended cylindrical indenters, seven flat-ended cylindrical indenters and to the more complex configuration of twelve randomly positioned indenters of arbitrary shapes: spherical-ended cylindrical, flat-ended cylindrical, conical and cylindrical indenters. The results will be discussed before concluding remarks.

\section{Algorithm for viscoelastic contacts}

We consider a rigid indenter of arbitrary shape $z(x, y)$ being pressed into contact with a viscoelastic solid. Under the action of a normal force the penetration of the indenter $\delta(t)$ and the contact areas will both grow with time and the distribution of contact pressure $p(x, y, t)$ will change. We wish to find the relations which link the pressure distribution, the resultant force $F(t)$ on the indenter and the penetration on the assumption that the surfaces are frictionless. Using Cartesian coordinates, the surface of the viscoelastic half-space before loading corresponds to the $(x, y)$ plane, as illustrated in Fig. 1 . The boundary conditions $(z=0)$ of our problem are given by

$$
\left\{\begin{array}{l}
u_{z}(x, y, t)=\delta(t)-|z(x, y)|, \quad(x, y) \in \Omega(t) \\
\sigma_{y z}=\sigma_{z z}=\sigma_{z x}=0, \quad(x, y) \notin \Omega(t)
\end{array}\right.
$$

where $u_{z}(x, y, t)$ is the normal component of the displacement of the point on the surface of the half-space, $\sigma_{i j}$ is the stress tensor, $\Omega(t)=\bigcup_{r=1}^{M} \Omega_{r}(t)$ is the current contact area, $M$ is the maximum number of contact areas (some $\Omega_{r}(t)$ may be empty). 
The Boussinesq's problem [2], involving the normal displacement of the point $(x, y)$ on the surface of the half-space, due to the normal load, for the elastic case may be written as:

$$
u_{z}(x, y)=\frac{(1-\nu)}{\pi} \frac{1}{2 \mu_{e}} \frac{p}{\rho}, \quad \rho=\sqrt{(x-\xi)^{2}+(y-\eta)^{2}}
$$

where $\mu_{e}$ is the elastic shear modulus, $\nu$ is the Poisson's ratio which has been taken as a constant. The analogue problem for the viscoelastic case [1] with the boundary condition may be written as:

$$
\begin{aligned}
u_{z}(x, y, t) & =\frac{(1-\nu)}{\pi} \int_{0}^{t} J(t-\tau)\left[\iint_{\Omega^{m}} \frac{1}{\rho} \frac{d p(\xi, \eta, \tau)}{d \tau} d \xi d \eta\right] d \tau \\
& =\delta(t)-|z(x, y)|, \quad(x, y) \in \Omega(t)
\end{aligned}
$$

where $\Omega^{m}=\bigcup_{r=1}^{M} \Omega_{r}^{m}$ is the maximum contact area. This domain is larger than the current contact area $\Omega(t)$ at each time step. Then the pressures in $\Omega^{m}$ are positive or null depending on whether the points are in contact or not at the considered time step. This problem may be solved numerically by using the Matrix Inversion Method (MIM), extended to viscoelastic cases [1]. But when the problem involves a large number of points the MIM can become very timeconsuming. Here the problem is solved using an alternative scheme, called the Two-scale Iterative Method (TIM), extended to viscoelastic cases. The normal component of the displacement $\left.u_{z}(x, y, t)\right|_{(x, y) \in \Omega_{r}(t)}$ has two components: $v_{r}$, which corresponds to the normal displacement of the point $(x, y)$ of the halfspace on the $r^{\text {th }}$ asperity because of the pressure in the $r^{\text {th }}$ asperity and $w_{r}$, which corresponds to the normal displacement of the point $(x, y)$ of the half- 
space on the $r^{\text {th }}$ asperity because of the pressures in the others asperities:

$$
\left\{\begin{array}{l}
\left.u_{z}(x, y, t)\right|_{(x, y) \in \Omega_{r}(t)}=v_{r}(x, y, t)+w_{r}(x, y, t)=\delta(t)-|z(x, y)| \\
v_{r}(x, y, t)=\frac{(1-\nu)}{\pi} \int_{0}^{t} J(t-\tau)\left[\iint_{\Omega_{r}^{m}} \frac{1}{\rho} \frac{d p(\xi, \eta, \tau)}{d \tau} d \xi d \eta\right] d \tau \\
w_{r}(x, y, t)=\frac{(1-\nu)}{\pi} \int_{0}^{t} J(t-\tau)\left[\int_{\Omega^{m} \backslash \Omega_{r}^{m}} \frac{1}{\rho} \frac{d p(\xi, \eta, \tau)}{d \tau} d \xi d \eta\right] d \tau \\
(x, y) \in \Omega_{r}(t), r=\overline{1, M}
\end{array}\right.
$$

Now our problem may be represented as follows:

$$
\begin{aligned}
& \frac{(1-\nu)}{\pi} \int_{0}^{t} J(t-\tau)\left[\iint_{\Omega_{r}^{m}} \frac{1}{\rho} \frac{d p(\xi, \eta, \tau)}{d \tau} d \xi d \eta\right] d \tau \\
& =v_{r}(x, y, t)=\delta(t)-|z(x, y)|-w_{r}(x, y, t) \\
& =\delta(t)-|z(x, y)|-\frac{(1-\nu)}{\pi} \int_{0}^{t} J(t-\tau)\left[\int_{\Omega^{m} \backslash \Omega_{r}^{m}} \frac{1}{\rho} \frac{d p(\xi, \eta, \tau)}{d \tau} d \xi d \eta\right] d \tau \\
& (x, y) \in \Omega_{r}(t), \quad r=\overline{1, M}
\end{aligned}
$$

The total force at point $r$ is given by:

$$
F_{r}(t)=\iint_{\Omega_{r}(t)} p(\xi, \eta, t) d \xi d \eta, \quad(x, y) \in \Omega_{r}(t), \quad r=\overline{1, M}
$$

Now the distribution of pressure for points different of $r$ is replaced by the concentrated normal forces. This is an approximation of our problem, which allows to accelerate the calculation. This yields:

$$
\begin{aligned}
w_{r}\left(X_{r}, Y_{r}, t\right) & =\frac{(1-\nu)}{\pi} \int_{0}^{t} J(t-\tau)\left[\iint_{\Omega^{m} \backslash \Omega_{r}^{m}} \frac{1}{\rho} \frac{d p(\xi, \eta, \tau)}{d \tau} d \xi d \eta\right] d \tau \\
& \approx \frac{(1-\nu)}{\pi} \sum_{\substack{s=1 \\
s \neq r}}^{M} \frac{1}{\rho_{r s}} \int_{0}^{t} J(t-\tau) \frac{d F_{s}(\tau)}{d \tau} d \tau, \quad r=\overline{1, M}
\end{aligned}
$$


where $\left(X_{r}, Y_{r}\right)$ are the coordinates of the tip of the $r^{\text {th }}$ asperity, $\quad \rho_{r s}=$ $\sqrt{\left(X_{r}-X_{s}\right)^{2}+\left(Y_{r}-Y_{s}\right)^{2}}$. In this case the problem may be represented as follows:

$$
\left\{\begin{array}{l}
\frac{(1-\nu)}{\pi} \int_{0}^{t} J(t-\tau)\left[\iint_{\Omega_{r}^{m}} \frac{1}{\rho} \frac{d p(\xi, \eta, \tau)}{d \tau} d \xi d \eta\right] d \tau \\
=\delta(t)-|z(x, y)|-\frac{(1-\nu)}{\pi} \sum_{\substack{s=1 \\
s \neq r}}^{M} \frac{1}{\rho_{r s}} \int_{0}^{t} J(t-\tau) \frac{d F_{s}(\tau)}{d \tau} d \tau \\
F_{r}(t)=\iint_{\Omega_{r}(t)} p(\xi, \eta, t) d \xi d \eta, \quad(x, y) \in \Omega_{r}(t), \quad r=\overline{1, M}
\end{array}\right.
$$

A surface of the half-space of size $L_{x} \times L_{y}$ was meshed using $N=n_{x} n_{y}$ rectangular elements of dimensions $d x=\frac{L_{x}}{n_{x}}$ and $d y=\frac{L_{y}}{n_{y}}$ with centres in $\left(x_{i}, y_{i}\right)$ and with uniform pressures acting on each of them (Fig. 2). The time discretization is $\tau=n_{\tau} d \tau$.

\section{Algorithm 1}

We will discretize the equations (5) and (6) in the form:

$$
\left\{\begin{array}{l}
\sum_{k=1}^{l} J((l-k) d \tau) \sum_{j=1}^{n_{r}} T_{i j} \frac{p_{j}^{k}-p_{j}^{k-1}}{d \tau} d \tau=\delta^{l}-z_{i} \\
-\sum_{\substack{s=1 \\
s \neq r}}^{M} \sum_{k=1}^{l} J((l-k) d \tau) \sum_{j=1}^{n_{s}} T_{i j} \frac{p_{j}^{k}-p_{j}^{k-1}}{d \tau} d \tau, \quad i=\overline{1, n_{r}} \\
F_{r}^{l}=\sum_{j=1}^{n_{r}} p_{j}^{l} d x d y, \quad r=\overline{1, M}
\end{array}\right.
$$

where $p_{j}^{k}=p\left(x_{j}, y_{j}, k d \tau\right), F_{r}^{l}=F_{r}(l d \tau), \delta^{l}=\delta(l d \tau), z_{i}=z\left(x_{i}, y_{i}\right)$ and $n_{r}=$ $n_{r}(t)$ is the number of contact elements for the $r^{t h}$ asperity for the time $t$. The influence coefficient $T_{i j}$, which expresses the displacement at a general point $i$ due to a unit pressure element centred at point $j$, was calculated using Love's 
results [39] for a rectangular surface acting on an elastic half-space

$$
T_{i j}=\frac{(1-\nu)}{\pi} \int_{y_{j}-\frac{d y}{2}}^{y_{j}+\frac{d y}{2} x_{j}-\frac{d x}{2}} \int_{\sqrt{2}}^{\frac{d x}{2}} \frac{d \xi d \eta}{\sqrt{\left(x_{i}-\xi\right)^{2}+\left(y_{i}-\eta\right)^{2}}}
$$

Then the first equation of the system (9) is written in the form:

$$
\left\{\begin{array}{l}
J(0) \sum_{j=1}^{n_{r}} T_{i j} p_{j}^{l}=\delta^{l}-z_{i}-v_{i}^{l-1} \\
-\sum_{\substack{s=1 \\
s \neq r}}^{M}\left[\sum_{k=1}^{l-1} J((l-k) d \tau) \sum_{j=1}^{n_{s}} T_{i j} \frac{p_{j}^{k}-p_{j}^{k-1}}{d \tau} d \tau+J(0) \sum_{j=1}^{n_{s}} T_{i j} \frac{p_{j}^{l}-p_{j}^{l-1}}{d \tau} d \tau\right], \\
v_{i}^{l-1}=\sum_{k=1}^{l-1} J((l-k) d \tau) \sum_{j=1}^{n_{r}} T_{i j} \frac{p_{j}^{k}-p_{j}^{k-1}}{d \tau} d \tau-J(0) \sum_{j=1}^{n_{r}} T_{i j} p_{j}^{l-1}, r=\overline{1, M}, i=\overline{1, n_{r}}
\end{array}\right.
$$

Then an iterative scheme for each moment of time $l$ is used for the calculation of normal contact pressure on each asperity. The first iteration:

$$
\begin{aligned}
& J(0) \sum_{j=1}^{n_{r}} T_{i j} p_{\mathbf{I} j}^{l}=\delta^{l}-z_{i}-v_{i}^{l-1}-\sum_{\substack{s=1 \\
s \neq r}}^{M} \sum_{k=1}^{l-1} J((l-k) d \tau) \sum_{j=1}^{n_{s}} T_{i j} \frac{p_{j}^{k}-p_{j}^{k-1}}{d \tau} d \tau \\
& r=\overline{1, M}, \quad i=\overline{1, n_{r}}
\end{aligned}
$$

Now it is possible to determine $p_{\mathbf{I} j}^{l}, j=\overline{1, n_{r}}, r=\overline{1, M}$ for a given history of loadings using the MIM which was programmed following the algorithm described in [37]. According to this algorithm, after solving the first equation of (12), some values of $p_{\mathbf{I} j}^{l}$ may be negative and for the next iteration of MIM these mesh points are excluded from the assumed contact area and the pressure 
there is taken to be zero. The second iteration of our iterative scheme:

$$
\begin{aligned}
& J(0) \sum_{j=1}^{n_{r}} T_{i j} p_{\mathbf{I I} j}^{l}=\delta^{l}-z_{i}-v_{i}^{l-1} \\
& -\sum_{\substack{s=1 \\
s \neq r}}^{M}\left[\sum_{k=1}^{l-1} J((l-k) d \tau) \sum_{j=1}^{n_{s}} T_{i j} \frac{p_{j}^{k}-p_{j}^{k-1}}{d \tau} d \tau+J(0) \sum_{j=1}^{n_{s}} T_{i j} \frac{p_{\mathbf{I} j}^{l}-p_{j}^{l-1}}{d \tau} d \tau\right] \\
& r=\overline{1, M}, \quad i=\overline{1, n_{r}}
\end{aligned}
$$

Now it is possible to determine $p_{\mathbf{I I} j}^{l}, j=\overline{1, n_{r}}, r=\overline{1, M}$. The iterative scheme is stopped when the following convergence criteria is respected:

$$
\frac{\sum_{j=1}^{n_{r}}\left(p_{\mathbf{N}+\mathbf{1} j}^{l}-p_{\mathbf{N} j}^{l}\right)^{2}}{\sum_{j=1}^{n_{r}}\left(p_{\mathbf{N} j}^{l}\right)^{2}}<\varepsilon, \quad r=\overline{1, M}
$$

The convergence parameter $\varepsilon$ is chosen by the operator and fixes the precision of the method. Then using the second equation of the system (9) it is possible to determine $F_{r}^{l}$.

Here it is necessary to take note of a correct comparison of $p_{j}^{k}$ with $p_{j}^{k-1}$ since the number of contact elements is different for times $k$ and $k-1$. For solving this problem one can introduce the matrix $\mathbf{T}_{\mathbf{r}}$ of dimension $n_{r}^{m} \times n_{r}^{m}$ and the vectors $\bar{p}_{r}$ of dimension $n_{r}^{m}, r=\overline{1, M}$ (the maximum number of contact elements, corresponding to $\left.\Omega_{r}^{m}\right)$.

Remark. The problem of multi-indentation on a viscoelastic half-space by an asperity of arbitrary shape may be solved using MIM which was extended to 
the viscoelastic problem in [1]:

$$
\left\{\begin{array}{l}
J(0) \sum_{j=1}^{n} T_{i j} p_{j}^{l}=\delta^{l}-z_{i}-v_{i}^{l-1}, \quad i=1 \ldots n \\
v_{i}^{l-1}=\sum_{k=1}^{l-1} J((l-k) d \tau) \sum_{j=1}^{n} T_{i j} \frac{p_{j}^{k}-p_{j}^{k-1}}{d \tau} d \tau-J(0) \sum_{j=1}^{n} T_{i j} p_{j}^{l-1}
\end{array}\right.
$$

where $n=\sum_{r=1}^{M} n_{r}$ is the number of contact elements for the time $t$ for all indenters. But when the problem involves a large number of points the MIM can become very time-consuming.

\section{Algorithm 2}

We will represent the system (8) in the form:

$$
\left\{\begin{array}{l}
\sum_{k=1}^{l} J((l-k) d \tau) \sum_{j=1}^{n_{r}} T_{i j} \frac{p_{j}^{k}-p_{j}^{k-1}}{d \tau} d \tau=\delta^{l}-z_{i} \\
-\frac{(1-\nu)}{\pi} \sum_{\substack{s=1 \\
s \neq r}}^{M} \frac{1}{\rho_{r s}} \sum_{k=1}^{l} J((l-k) d \tau) \frac{F_{s}^{k}-F_{s}^{k-1}}{d \tau} d \tau, \quad i=\overline{1, n_{r}} \\
F_{r}^{l}=\sum_{j=1}^{n_{r}} p_{j}^{l} d x d y, \quad r=\overline{1, M}
\end{array}\right.
$$

Then the system (16) is written as follows:

$$
\left\{\begin{array}{l}
J(0) \sum_{j=1}^{n_{r}} T_{i j} p_{j}^{l}=\delta^{l}-z_{i}-v_{i}^{l-1} \\
-\frac{(1-\nu)}{\pi} \sum_{\substack{s=1 \\
s \neq r}}^{M} \frac{1}{\rho_{r s}}\left[\sum_{k=1}^{l-1} J((l-k) d \tau) \frac{F_{s}^{k}-F_{s}^{k-1}}{d \tau} d \tau+J(0) \frac{F_{s}^{l}-F_{s}^{l-1}}{d \tau} d \tau\right] \\
v_{i}^{l-1}=\sum_{k=1}^{l-1} J((l-k) d \tau) \sum_{j=1}^{n_{r}} T_{i j} \frac{p_{j}^{k}-p_{j}^{k-1}}{d \tau} d \tau-J(0) \sum_{j=1}^{n_{r}} T_{i j} p_{j}^{l-1} \\
F_{r}^{l}=\sum_{j=1}^{n_{r}} p_{j}^{l} d x d y, \quad i=\overline{1, n_{r}}, \quad r=\overline{1, M}
\end{array}\right.
$$

Then an iterative scheme for each moment of time $l$ is used for the calculation 
of the normal contact pressure on each asperity. The first iteration is:

$$
\left\{\begin{array}{l}
J(0) \sum_{j=1}^{n_{r}} T_{i j} p_{\mathbf{I} j}^{l}=\delta^{l}-z_{i}-v_{i}^{l-1} \\
-\frac{(1-\nu)}{\pi} \sum_{\substack{s=1 \\
s \neq r}}^{M} \frac{1}{\rho_{r s}} \sum_{k=1}^{l-1} J((l-k) d \tau) \frac{F_{s}^{k}-F_{s}^{k-1}}{d \tau} d \tau, \quad i=\overline{1, n_{r}} \\
F_{\mathbf{I} r}^{l}=\sum_{j=1}^{n_{r}} p_{\mathbf{I} j}^{l} d x d y, \quad r=\overline{1, M}
\end{array}\right.
$$

Now it is possible to determine $p_{\mathbf{I} j}^{l}, j=\overline{1, n_{r}}, r=\overline{1, M}$ for a given history of loadings. Then using the second equation of the system (18) it is possible to determine $F_{\mathbf{I} r}^{l}$. The second iteration of our iterative scheme:

$$
\left\{\begin{array}{l}
J(0) \sum_{j=1}^{n_{r}} T_{i j} p_{\mathbf{I I} j}^{l}=\delta^{l}-z_{i}-v_{i}^{l-1} \\
-\frac{(1-\nu)}{\pi} \sum_{\substack{s=1 \\
s \neq r}}^{M} \frac{1}{\rho_{r s}}\left[\sum_{k=1}^{l-1} J((l-k) d \tau) \frac{F_{s}^{k}-F_{s}^{k-1}}{d \tau} d \tau+J(0) \frac{F_{\mathbf{I} s}^{l}-F_{s}^{l-1}}{d \tau} d \tau\right], i=\overline{1, n_{r}} \\
F_{\mathbf{I I} r}^{l}=\sum_{j=1}^{n_{r}} p_{\mathbf{I I} j}^{l} d x d y, \quad r=\overline{1, M}
\end{array}\right.
$$

Now it is possible to determine $p_{\mathbf{I I} j}^{l}, F_{\mathbf{I I} r}^{l}, j=\overline{1, n_{r}}, r=\overline{1, M}$. The iterative scheme is stopped when the convergence criteria (14) is respected.

\section{$3 \quad$ Numerical examples}

Five geometries of indenters will be studied as shown in Fig. 3: spherical indenter of radius $R$, spherical-ended cylindrical indenter which is composed of a cylindrical base of radius $a$ and a spherical end of radius $R>a$, flatended cylindrical indenter of radius $a$, conical indenter of semi-opening angle $\theta$, cylindrical indenter of radius $R$ and of width $b$ (finite cylindrical shape with 
its curved face).

In the following examples the stress history is represented in the form of a Prony's series:

$$
\sigma(t)=\left(E_{\infty}+\left(E_{0}-E_{\infty}\right) e^{-\frac{t}{\tau_{c}}}\right) \varepsilon_{0}
$$

where $\tau_{c}$ is the characteristic time, $E_{0}$ is the instantaneous Young's modulus, $E_{\infty}$ is the statical Young's modulus, $\varepsilon_{0}$ is the instantaneous strain. Then the creep and the relaxation functions may be written as follows:

$$
\left\{\begin{array}{l}
G(t)=E_{\infty}+\left(E_{0}-E_{\infty}\right) e^{-\frac{t}{\tau_{c}}} \\
J(t)=\frac{1}{E_{\infty}}-\frac{\left(E_{0}-E_{\infty}\right)}{E_{0} E_{\infty}} e^{-\frac{E_{\infty}}{E_{0}} \frac{t}{\tau_{c}}}
\end{array}\right.
$$

Now for the numerical calculations we take the following parameter values $E_{\infty}=7.5 \mathrm{MPa}, E_{0}=15 \mathrm{MPa}, \tau_{c}=1 \mathrm{~s}$. The penetration $\delta(t)=\sin \left(\frac{\pi t}{L_{t}}\right)$ is imposed with the duration of loading $L_{t}=5 \mathrm{~s}$, so that the penetration $\delta(t)$ increases monotonically to $1 \mathrm{~mm}$ and then decreases monotonically to $0 \mathrm{~mm}$ Fig. 5 (b).

\subsection{The indentation of a viscoelastic half-space by seven rigid spherical in- denters}

This configuration is composed of seven rigid spherical indenters of the type shown in Fig. 3 (a). The indenters are identical with radius $R=5 \mathrm{~mm}$. Fig. 4 shows the upper view of this configuration with $L_{x}=L_{y}=30 \mathrm{~mm}$. The 
coordinates of their tips are:

$$
\begin{array}{ll}
x_{1}=0, & y_{1}=0, \\
x_{2}=0, & y_{2}=2 R, \\
x_{3}=\sqrt{3} R, & y_{3}=R, \\
x_{4}=\sqrt{3} R, & y_{4}=-R, \\
x_{5}=0, & y_{5}=-2 R, \\
x_{6}=-\sqrt{3} R, & y_{6}=-R, \\
x_{7}=-\sqrt{3} R, & y_{7}=R
\end{array}
$$

A comparison between the contact forces obtained with Algorithm 2 for a central and a peripheral indenters with $n_{\tau}=401, n_{x}=n_{y}=91$ and the convergence parameter $\varepsilon=0.1$ (which is chosen and will not be changed) is made in Fig. 5 (a). The difference between the two curves is large. The reason is the influence of the peripheral indenters on the central indenter. The peripheral indenters deform the viscoelastic half-space under the central indenter and the contact force for the central indenter is less than for the peripheral indenters. Also one can see the asymmetry between increasing and decreasing loadings of the viscoelastic curves. The contact forces may equal zero even if the penetration does not yet equal zero. The reason is the memory of the viscoelastic material.

In Fig. 6 the dependence of forces versus the penetration is illustrated. As previously, the difference between the two curves is large. Also here hysteresis is observed in the force-penetration curve as viscoelastic substance loses energy 
when a load is increasing, then decreasing.

The distribution of the pressure $p(x, y, t)$ and the pressure $p(0, y, t)$ for $t=2.5$ s are represented in Fig. 7 with $n_{\tau}=401, n_{x}=n_{y}=201$. For each indenter this is the analogue of the Hertz's distribution for the viscoelastic case. As previously, the influence of the peripheral indenters on the central indenter is observed. The peripheral contact areas are larger than the central contact area and the absolute value of pressure for the peripheral indenter is higher than for the central indenter.

A comparison between the contact forces obtained with Algorithm 1 and Algorithm 2 with $n_{\tau}=401, n_{x}=n_{y}=91$ is made in Fig. 8. The difference between the solutions is small, showing a very good agreement. Since Algorithm 2 is about five times faster than Algorithm 1, common sense guides us to use it.

The TIM was validated by comparing to the Finite Elements Method (FEM) and to the MIM for the elastic case using contact force results. The Young's modulus has been taken equal $10 \mathrm{MPa}, R=10 \mathrm{~mm}, n_{x}=n_{y}=121$. The penetration was varied between 0 and $2 \mathrm{~mm}$. The FEM analysis was done using the bi-potential method [40]. The simulation of the seven spherical indenters in contact with the elastic half-space required a three-dimensional FE model. Due to symmetry, only one twelfth of the initial sample was considered. The halfspace was subdivided into 12000 eight-node brick-like elements. The indenters were modelled with 408 rigid shell elements. Contact elements were generated between the shell and the top surface of the half-space so as to prevent an interpenetration of the contact surfaces. A vertical rigid motion was imposed on the indenters by an amount of $2 \mathrm{~mm}$. Newton-Raphson iterative algorithm 
was used and the displacement was applied in 20 load steps. The results of the TIM and the FEM are compared for the central and the peripheral indenters in Fig. 9. The method MIM has been taken as a reference. The MIM and the TIM provide very similar results (the relative difference is less than $2 \%$ ). Thus, the interaction between the indenters is well taken into account by the TIM. The FEM leads to much larger errors which are more than $12 \%$ for the peripheral indenters and close to $20 \%$ for the central indenter. Although it is possible to improve the accuracy of the results by using a finer mesh in the FEM, the calculations will become excessively time consuming.

The TIM was also compared to the measured data for this configuration (elastic half-space, seven rigid spherical indenters) in the work of Cesbron et al. [41]. The differences between experiments and numerical results were below $10 \%$.

\subsection{The indentation of a viscoelastic half-space by seven rigid spherical-ended cylindrical indenters}

This configuration is composed of seven identical rigid spherical-ended cylindrical indenters of the type shown in Fig. 3 (b) of radius $R=10 \mathrm{~mm}$ and base radius $a$. We consider two cases with the different values of base radius $a=2.5 \mathrm{~mm}$ and $a=3.5 \mathrm{~mm}$. Spherical-ended cylindrical indenters are used to get large and close contact areas. For the spherical case when the radius $R$ is large the contact areas are also large but not close. The coordinates of the tips of the indenters are the same as relations (22) with replacement of $R$ by $a$. Thus, the configuration with $a=2.5 \mathrm{~mm}$ correspond to closer cases. 
The distribution of the pressure $p(x, y, t)$ and the pressure $p(0, y, t)$ obtained with Algorithm 2 for $a=2.5 \mathrm{~mm}, t=1 \mathrm{~s}$ are represented in Fig. 10 with $n_{\tau}=401, n_{x}=n_{y}=201$. The influence of the peripheral indenters on the central indenter is stronger than for the spherical case because the contact areas are very close. For this case the contact areas for peripheral indenters are much larger than for the central indenter and the absolute value of pressure for the peripheral indenter is much higher than for the central indenter.

The distribution of the pressure $p(x, y, t)$ and the pressure $p(0, y, t)$ obtained with Algorithm 1 for $a=3.5 \mathrm{~mm}, t=1 \mathrm{~s}$ are represented in Fig. 11 with $n_{\tau}=401, n_{x}=n_{y}=121$. Under the influence of the central indenter the maximum value of pressure shifts outside and no more in the tip of the peripheral indenter. Thus, one can see the asymmetry of the distribution of the pressure for peripheral indenters.

A comparison between the contact forces obtained with Algorithm 1 and Algorithm 2 for the base radius $a=3.5 \mathrm{~mm}$ with $n_{\tau}=401, n_{x}=n_{y}=61$ is made in Fig. 12 (a). The difference between the solutions is small, showing a good agreement, but higher than for the spherical case when the contact areas are not close. A comparison between the distributions of the pressure obtained with Algorithm 1 and Algorithm 2 for $t=1 \mathrm{~s}$ with $n_{\tau}=401, n_{x}=n_{y}=121$ is made in Fig. 12 (b). This difference between the solutions is also small, showing a good agreement. Yet one can see that Algorithm 1 catches better the influence of the central indenter on the peripheral indenters.

The TIM can be also validated by comparing the numerical results to the numerical results obtained with MIM which were also compared to the analytic solutions in cases of a single spherical asperity (loading and unloading) and a 
single conical asperity (loading only) in the work [1]. The comparison is made in Fig. 13 for $n_{\tau}=401, n_{x}=n_{y}=61$ and $a=3.5 \mathrm{~mm}$. The total contact force computed by the TIM agrees very well with the total contact force computed by the MIM. But for the chosen parameters the TIM is about eight times faster than the MIM.

\subsection{The indentation of a viscoelastic half-space by seven rigid flat-ended cylindrical indenters}

This configuration is composed of seven rigid flat-ended cylindrical indenters of the type shown in Fig. 3 (c) of base radius $a=1 \mathrm{~mm}$. A comparison between the contact forces obtained with Algorithm 2 for a central and peripheral indenters with $n_{\tau}=401, n_{x}=n_{y}=201$ is made in Fig. 14 with the penetration $\delta(t)$ imposed in Fig. 5 (b), as for the spherical case. Again, the difference between the two curves is large. The reason is the same - the influence of the peripheral indenters on the central indenter.

In Fig. 15 the dependence of forces versus the penetration is illustrated. Also here, as for the spherical case, hysteresis is observed in the force-penetration curve as viscoelastic substance loses energy when a load is increasing, then decreasing.

The distribution of the pressure $p(x, y, t)$ and the pressure $p(x, 0.56 x, t)$ (the pressure distribution along a diagonal line) for $t=1 \mathrm{~s}$ are represented in Fig. 16 with $n_{\tau}=401, n_{x}=n_{y}=421$. The maximum pressure for each contact area is concentrated at the boundaries of contact areas and the value of pressure for the peripheral indenter is higher than for the central indenter. 
Yet the distribution of the pressure for peripheral indenters is not symmetric.

\author{
3.4 The indentation of a viscoelastic half-space by twelve rigid indenters of \\ arbitrary shape
}

\begin{abstract}
Now we consider the more general case of twelve indenters of arbitrary shapes.
This configuration is composed of three rigid spherical-ended cylindrical indenters of base radius $a=3 \mathrm{~mm}$ and radius $R=5,6,10 \mathrm{~mm}$, of three flat-ended cylindrical indenters of base radius $a=1,1,1.5 \mathrm{~mm}$, of three conical indenters
\end{abstract} of the type shown in Fig. $3(\mathrm{~d})$ of semi-opening angle $\theta=\frac{\pi}{4}$ and of three cylindrical indenters (finite cylindrical shape with its curved face) of the type shown in Fig. 3 (e) of radius $R=3 \mathrm{~mm}$ and of width $b=1 \mathrm{~mm} ; R=2$ $\mathrm{mm}, b=2 \mathrm{~mm} ; R=1 \mathrm{~mm}, b=3 \mathrm{~mm}$. This case is more complex since the indenting geometry doesn't have an axisymmetric profile and the positions of indenters are arbitrarily. The distribution of the pressure $p(x, y, t)$ and the pressure $p(0, y, t)$ obtained with Algorithm 1 for $t=1 \mathrm{~s}$ are represented in Fig. 17 with $n_{\tau}=401, n_{x}=n_{y}=161$. The maximum pressure for flat-ended cylindrical indenters is concentrated at the boundaries of each contact area. The maximum pressure for cylindrical indenters is concentrated at the boundaries of the contact area which are perpendicular to the axis $y$ which is the axis of symmetry of the cylinder. The maximum pressures for cylindrical and flat-ended cylindrical indenters are comparable and the distributions are not symmetric. The maximum pressure for spherical-ended cylindrical indenters is less than for the others indenters. The absolute maximum pressure is on the conical indenters, but the contact areas are the smallest. 


\section{Conclusions}

In this paper the Matrix Inversion Method (MIM) introduced by Kalker and used for the analysis of elastic and viscoelastic [1] contacts, was revised to the viscoelastic problem for indenters of arbitrary shape when a few contact areas are available. On the basis of this alternative scheme, called the Two-scale Iterative Method (TIM), two algorithms were proposed. With the aid of these algorithms the contact forces as a function of time, the pressure distribution, the contact area are found for a simple configuration of seven spherical indenters, seven spherical-ended cylindrical indenters, seven flat-ended cylindrical indenters as well as for a more complex configuration of twelve randomly positioned indenters of arbitrary shapes: spherical-ended cylindrical, flat-ended cylindrical, conical and cylindrical indenters (finite cylindrical shape with its curved face).

The TIM was validated by comparing the numerical results to the numerical results obtained with MIM in the case of a seven spherical-ended cylindrical indenters. The total contact force computed by the TIM agrees very well with the total contact force computed by the MIM.

For a simple configuration of seven spherical indenters one can see the difference between the forces for central and peripheral indenters. The peripheral indenters deform the viscoelastic half-space under the central indenter and the contact force for the central indenter is less than for the peripheral indenters. As consequence, the peripheral contact areas are larger than the central contact area and the absolute value of pressure for the peripheral indenter is higher than for the central indenter. The difference between the Algorithm 1 
and Algorithm 2 is small and since Algorithm 2 is about five times as fast as Algorithm 1, common sense guides us to use it.

For a simple configuration of seven spherical-ended indenters when the indenters are very close the difference between the Algorithm 1 and Algorithm 2 is also small, but higher than for the spherical case when the contact areas are not very close. For this case the Algorithm 1 catches better the influence of the central indenter on the peripheral indenters. Under the influence of the central indenter the maximum value of pressure shifts outside and is no more in the tip of the peripheral indenter. Here one can see the asymmetry of distribution of the pressure for peripheral indenters.

For a simple configuration of seven flat-ended cylindrical indenters the maximum pressure for each contact area is concentrated at the boundaries and the value of pressure for the peripheral indenter is higher than for the central indenter.

And the last example of twelve randomly positioned indenters of arbitrary shapes shows that the method proposed in this paper could be probably applied to practical contact problems with a large number of asperities, such as for tyre-road contact computations.

\section{Acknowledgements}

This work is supported by RFBR (08-01-00600, 08-08-00553). 


\section{References}

[1] Kozhevnikov IF, Cesbron J, Duhamel D, Yin HP, Anfosso-Lédée F. A new algorithm for computing the indentation of a rigid body of arbitrary shape on a viscoelastic half-space. International Journal of Mechanical Sciences 2008;50:11941202.

[2] Boussinesq J. Application des potentiels à l'étude de l'équilibre et du mouvement des solides élastiques. Gauthier-Villars, Paris, 1885.

[3] Hertz H. Über die Berührung fester elastischer Körper (On the contact of elastic solids). J. Reine und Angewandte Mathematik 1882;92:156-171.

[4] Hertz H. Über die Berührung fester elastischer Körper and über die Harte (On the contact of rigid elastic solids and on hardness). Verhandlungen des Vereins zur Beförderung des Gewerbefleisses, Leipzig 1882.

[5] Sneddon IN. The relation between load and penetration in the axisymmetric Boussinesq problem for a punch of arbitrary profile. International Journal of Engineering Science 1965;3:47-57.

[6] Vilke VG. On Non-Hertzian Contact of Wheel and Rail. Research on Problems of Stability and Stabilization of Motion, Reports of the Computing Centre of the Russian Ac. of Sc. 2007;137-157.

[7] Radok JRM. Viscoelastic stress analysis. Q. App. Math. 1957;15:198.

[8] Ting TCT. The contact stresses between a rigid indenter and a viscoelastic halfspace. Trans. ASME, Series E, Journal of Applied Mechanics 1966;33:845.

[9] Vandamme M, Ulm F-J. Viscoelastic solutions for conical indentation. International Journal of Solids and Structures 2006;43:3142-3165.

[10] Larsson P-L \& Carlsson S. On microindentation of viscoelastic polymers. Polymer Testing 1998;17:49-75. 
[11] Yang WH. The contact problem for viscoelastic bodies. Trans. ASME, Series E, Journal of Applied Mechanics 1966;33:395.

[12] Giannakopoulos AE. Elastic and viscoelastic indentation of flat surfaces by pyramid indentors. Journal of the Mechanics and Physics of Solids 2006;54:13051332.

[13] Murakami Y, Tanaka K, Itokazu M, Shimamoto A. Elastic analysis of triangular pyramid indentation by the finite element method and its application to nanoindentation measurement of glasses. Philos. Mag. 1994;A69:1131-1153.

[14] Larsson P-L, Giannakopoulos AE, Soderlund E, Rowcliffe DJ, Vestergaard R. Analysis of Berkovich indentation. Int. J. Solids Struct. 1996;33:221-248.

[15] Rabinovich VL, Savin VK. Three dimensional modelling of indentation fracture in brittle materials. Mat. Sci. Eng. 1996;A206:208-214.

[16] Cheng L, Xia X, Scriven LE, Gerberich WW. Spherical-tip indentation of viscoelastic material. Mechanics of Materials 2005;37:213-226.

[17] Greenwood JA, Williamson JBP. Contact of nominally flat surfaces. In: Proceedings of the Royal Society, London, 1966. Series A, 295.

[18] McCool J. Comparison of Models for the Contact of Rough Surfaces. Wear 1986;107:37-60.

[19] Carbone G, Bottiglione F. Asperity contact theories: Do they predict linearity between contact area and load? Journal of the Mechanics and Physics of Solids 2008;56:2555-2572.

[20] Kucharski S, Klimczak T, Polijaniuk A, Kaczmarek J. Finite-elements model for the contact of rough surfaces. Wear 1994;177:1-13.

[21] Adams GG, Nosonovsky M. Contact modeling - forces. Tribology International 2000;33:431-442. 
[22] Cesbron J, Anfosso-Lédée F, Duhamel D, Yin HP, Le Houédec D. Experimental study of tyre/road contact forces in rolling conditions for noise prediction. Journal of Sound and Vibration 2008; doi:10.1016/j.jsv.2008.07.018.

[23] Kragelsky IV, Demkin NB. Contact area of rough surfaces. Wear 1960;3:170187.

[24] O'Callaghan M, Cameron MA. Static contact under load between nominally flat surfaces in which deformation is purely elastic. Wear 1976;36.

[25] Bush AW, Gibson RD, Thomas TR. The elastic contact of a rough surface. Wear 1975;35:87-111.

[26] Singh KP, Paul B. Numerical Solution of Non-Hertzian Elastic Contact Problems. ASME Journal of Applied Mechanics 1974;96:484-490.

[27] Webster MN, Sayles RS. A Numerical Model for the Elastic Frictionless Contact of Real Rough Surfaces. ASME Journal of Tribology 1986;108:314-320.

[28] Ju Y, Zheng L. A full numerical solution for the elastic contact of threedimensional real rough surfaces. Wear 1992;157:151-161.

[29] Chang L, Gao Y. A Simple Numerical Method for Contact Analysis of Rough Surfaces. ASME Journal of Tribology 1999;121:425-432.

[30] Nayak L, Johnson KL. Pressure between elastic bodies having a slender area of contact and arbitrary profiles. International Journal of Mechanical Sciences 1979;21:237-247.

[31] Nowell D, Hills DA. Hertzian contact of ground surfaces. ASME Journal of Tribology 1989;111:175-179.

[32] Ciavarella M, Hills DA, Moobola R. Analysis of plane and rough contact, subject to a shearing force. International Journal of Mechanical Sciences 1999;41:107-120. 
[33] Karpenko YuA, Akay A. A numerical model of friction between rough surfaces. Tribology International 2001;34:531-545.

[34] Liu Z, Neville A, Reuben RL. A Numerical Calculation of the Contact Area and Pressure of Real Surfaces in Sliding Wear. Journal of Tribology 2001;123:27-35.

[35] Stanley HM, Kato T. An FFT-Based Method for Rough Surface Contact. ASME Journal of Tribology 1997;119:481-485.

[36] Kalker JJ. Two algorithms for the contact problem in elastostatics. Report of the Department of Mathematics and Informatics, 82-26. Delft University of Technology, 1982.

[37] Johnson KL. Contact Mechanics. Cambridge University Press, 1985.

[38] Cesbron J, Anfosso-Lédée F, Yin HP, Duhamel D, Le Houédec D. Influence of road texture on tyre/road contact in static conditions - Numerical and experimental comparison. Accepted for publication in the International Journal of Road Materials and Pavement Design.

[39] Love AEH. Stress produced in a semi-infinite solid by pressure on part of the boundary. In: Phil. Trans. Royal Society 1929;A228:37-43.

[40] De Saxcé G, Feng Z-Q. The bipotential method: A constructive approach to design the complete contact law with friction and improved numerical algorithms. Mathematical and Computer Modelling 1998;28:225-245.

[41] Cesbron J, Yin HP, Anfosso-Lédée F, Duhamel D, Le Houédec D, Feng ZQ. Numerical and experimental study of multi-contact on an elastic half-space. International Journal of Mechanical Sciences 2009;51:33-40. 


\section{List of Figures}

1 The contact between a rigid indenter of arbitrary shape and a viscoelastic half-space

2 The discretization of the contact problem by rectangular elements

3 The indenters used in the article, (a) the spherical indenter, (b) the spherical-ended cylindrical indenter, $(c)$ the flat-ended cylindrical indenter, $(d)$ the conical indenter, $(e)$ the cylindrical indenter

4 Upper view of the seven spherical indenters configuration

5 Spherical case. The penetration $\delta(t)$ is imposed $(a)$ The difference between the contact forces,- - central indenter, ----- peripheral indenter $(b)$ The penetration $\delta(t)$

6 Spherical case. The difference between the contact forces, central indenter, ------ peripheral indenter

$7 \quad$ Spherical case. At the top the pressure distribution for $t=2.5$ s. At the bottom the pressure for $x=0 \mathrm{~mm}$

8 Spherical case. The penetration $\delta(t)$ is imposed. The difference between the contact forces,_— central indenter (Algorithm 1), ----- peripheral indenter (Algorithm 1), …... central indenter (Algorithm 2), .......... peripheral indenter (Algorithm 2) 
9 Spherical case (elastic). The difference between the contact forces, + central indenter $(\mathrm{TIM}), \times$ peripheral indenter $(\mathrm{TIM})$, central indenter $(\mathrm{FEM}), \triangle$ peripheral indenter $(\mathrm{FEM})$

10 Spherical-ended cylindrical case. At the top the pressure distribution obtained with Algorithm 2 for $t=1 \mathrm{~s}$ and $a=2.5$ $\mathrm{mm}$. At the bottom the pressure for $x=0 \mathrm{~mm}$.

11 Spherical-ended cylindrical case. At the top the pressure distribution obtained with Algorithm 1 for $t=1 \mathrm{~s}$ and $a=3.5$ $\mathrm{mm}$. At the bottom the pressure for $x=0 \mathrm{~mm}$.

12 Spherical-ended cylindrical case. The penetration $\delta(t)$ is imposed. (a) The difference between the contact forces, — central indenter (Algorithm 1), ----- peripheral indenter (Algorithm 1), …... central indenter (Algorithm 2), …...... peripheral indenter (Algorithm 2) (b) The difference between the pressures for $t=1 \mathrm{~s}, x=0 \mathrm{~mm}$ and $a=3.5 \mathrm{~mm}, \square$ Algorithm 1, -----Algorithm 2

13 Spherical-ended cylindrical case. The penetration $\delta(t)$ is imposed. The difference between the contact forces, MIM, ------ TIM

14 Flat-ended cylindrical case. The penetration $\delta(t)$ is imposed.

The difference between the contact forces, _ central indenter, ------ peripheral indenter

15 Flat-ended cylindrical case. The difference between the contact forces, —— central indenter, ------ peripheral indenter 
16 Flat-ended cylindrical case. At the top the pressure distribution for $t=1 \mathrm{~s}$. At the bottom the pressure $p(x, 0.56 x, 1)$

17 General case: three spherical-ended cylindrical indenters, three flat-ended cylindrical indenters, three conical indenters, three cylindrical indenters. At the top the pressure distribution for $t=1 \mathrm{~s}$. At the bottom the pressure for $x=0 \mathrm{~mm}$. 

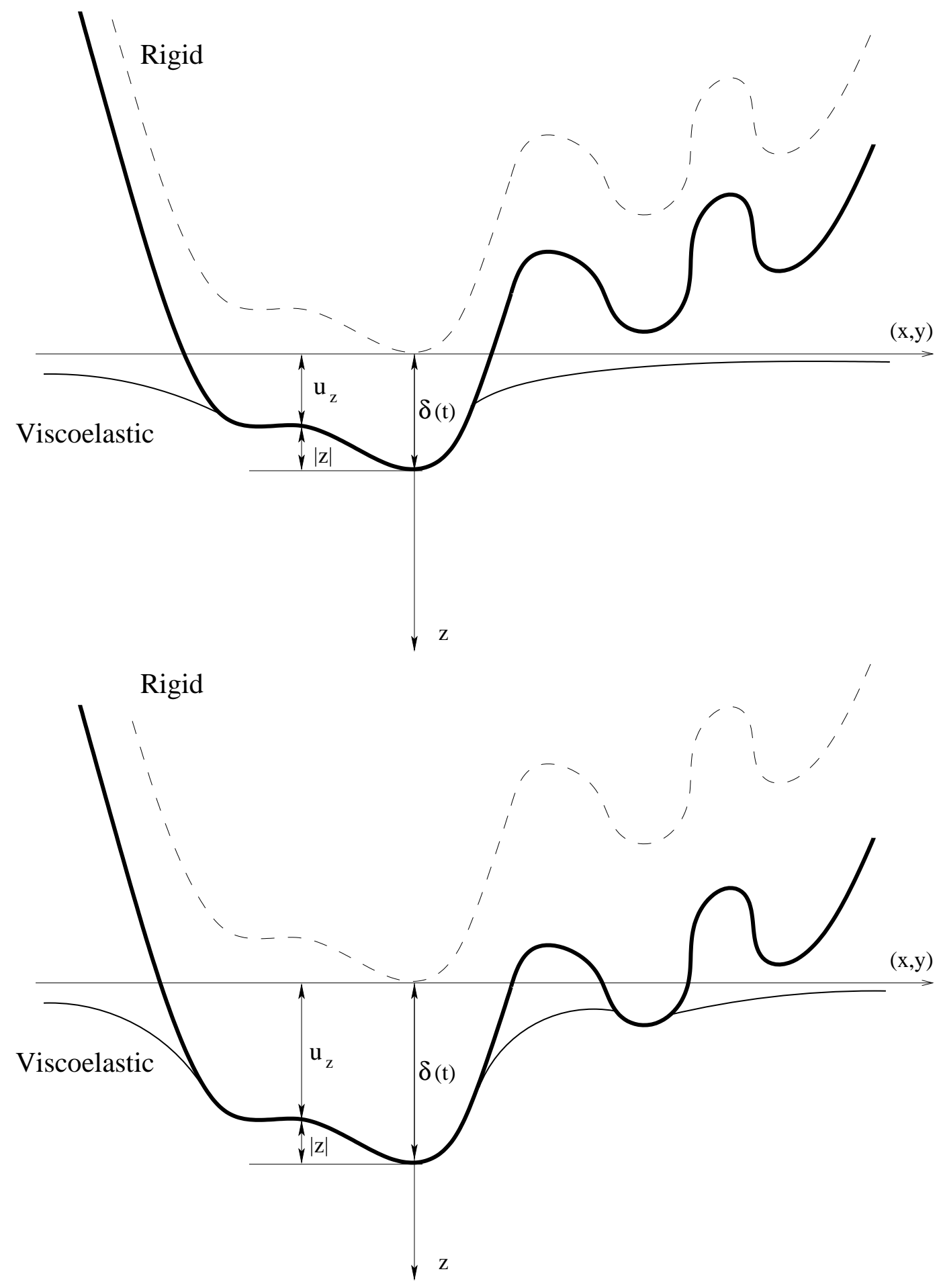

Fig. 1. The contact between a rigid indenter of arbitrary shape and a viscoelastic half-space 


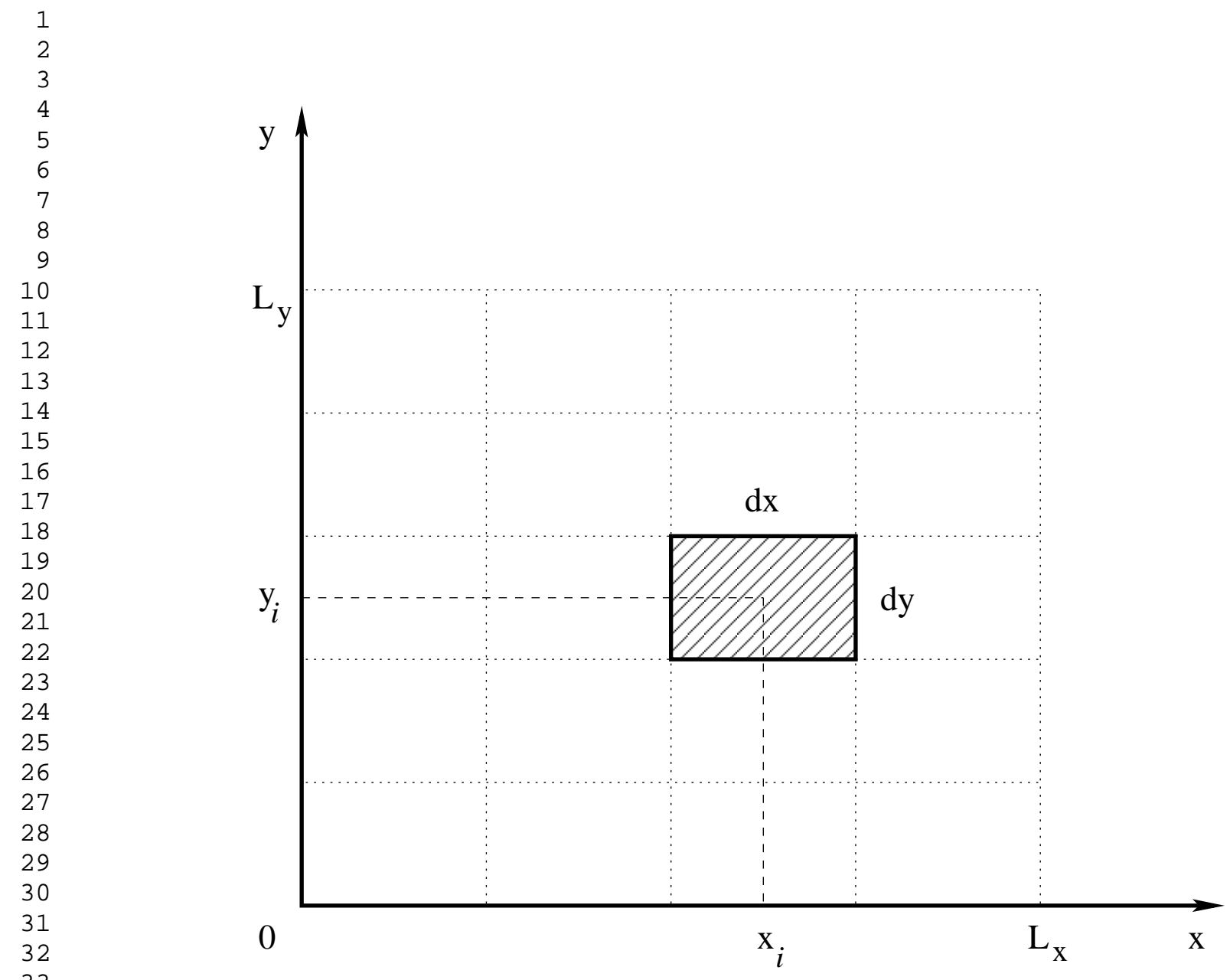

Fig. 2. The discretization of the contact problem by rectangular elements 
(a)

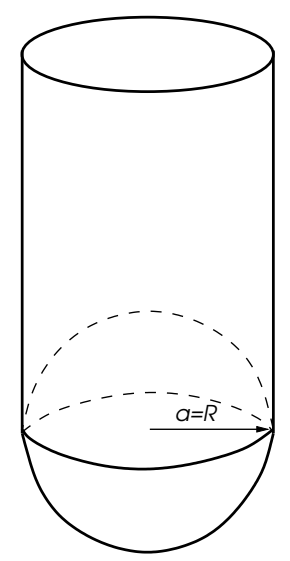

(b)

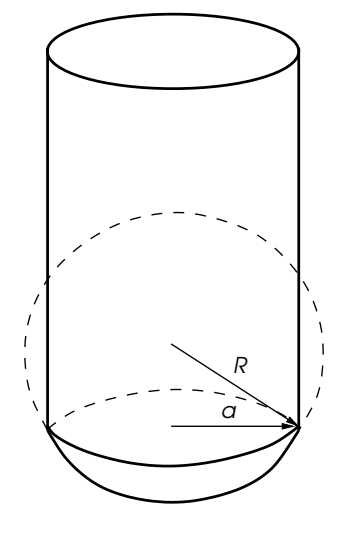

(c)

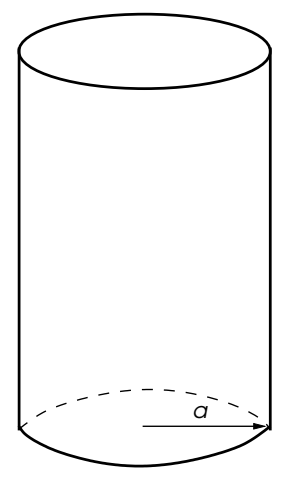

(d)

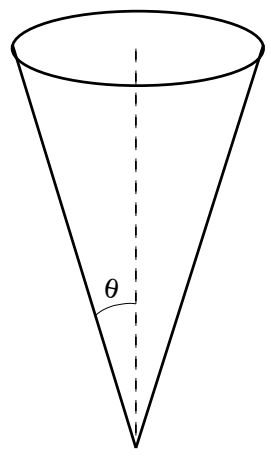

(e)

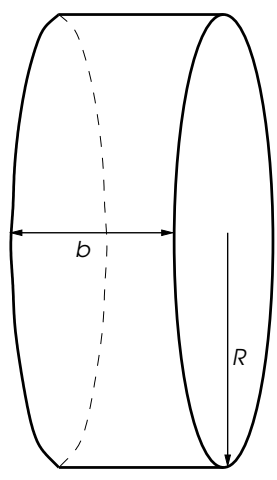

Fig. 3. The indenters used in the article, $(a)$ the spherical indenter, $(b)$ the spherical-ended cylindrical indenter, $(c)$ the flat-ended cylindrical indenter, $(d)$ the conical indenter, $(e)$ the cylindrical indenter 


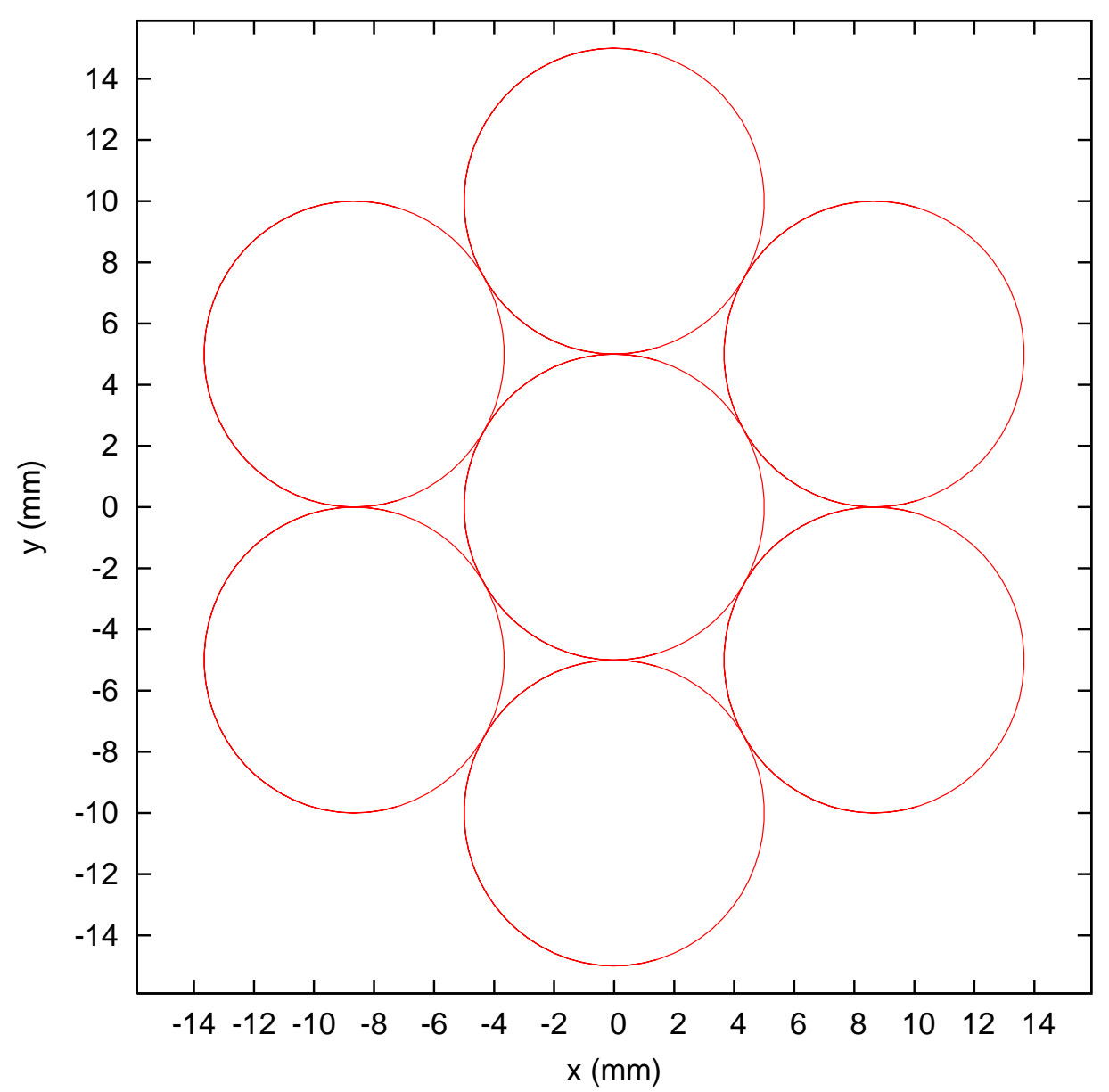

Fig. 4. Upper view of the seven spherical indenters configuration 
(a) Viscoelastic spherical case

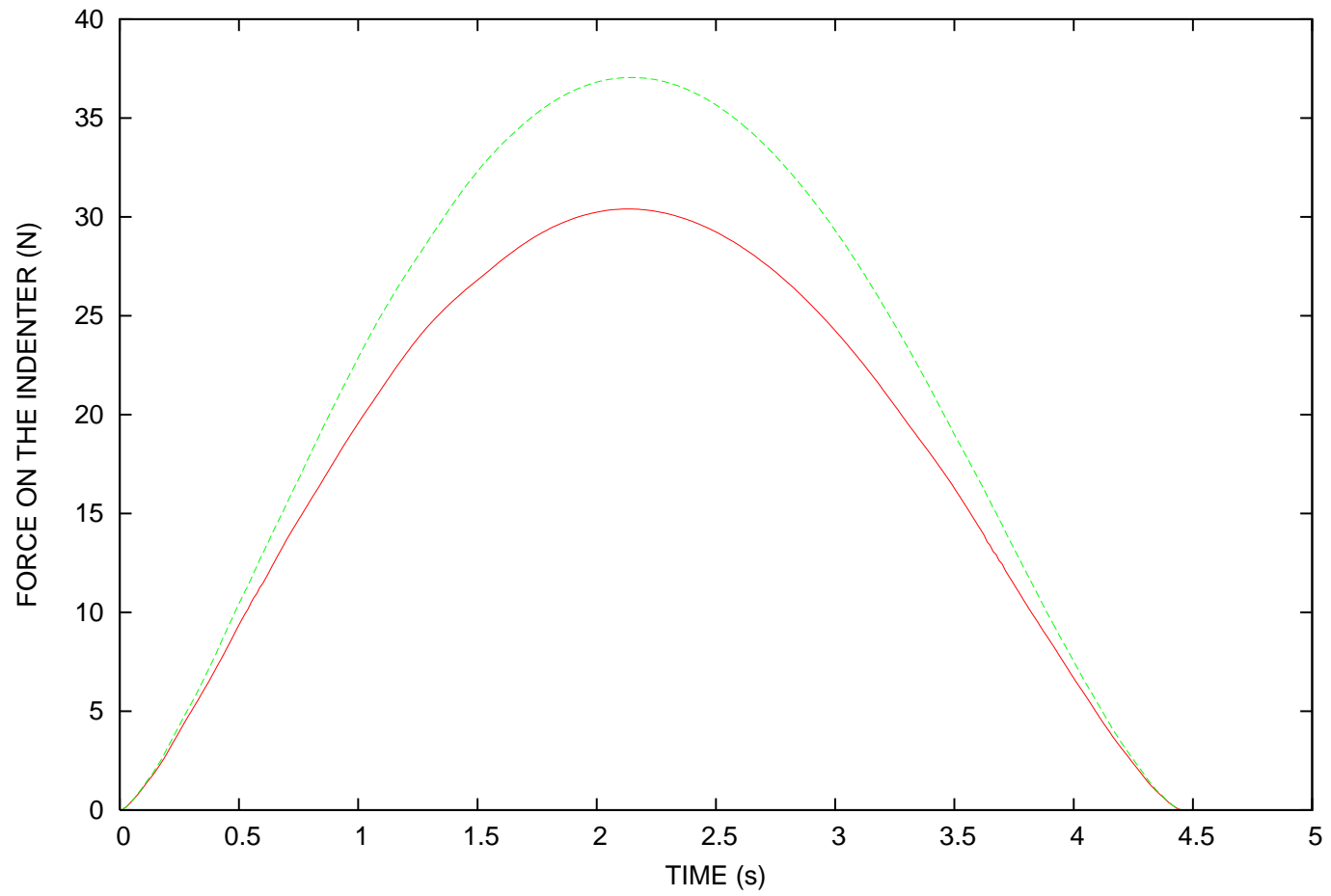

(b) Viscoelastic spherical case

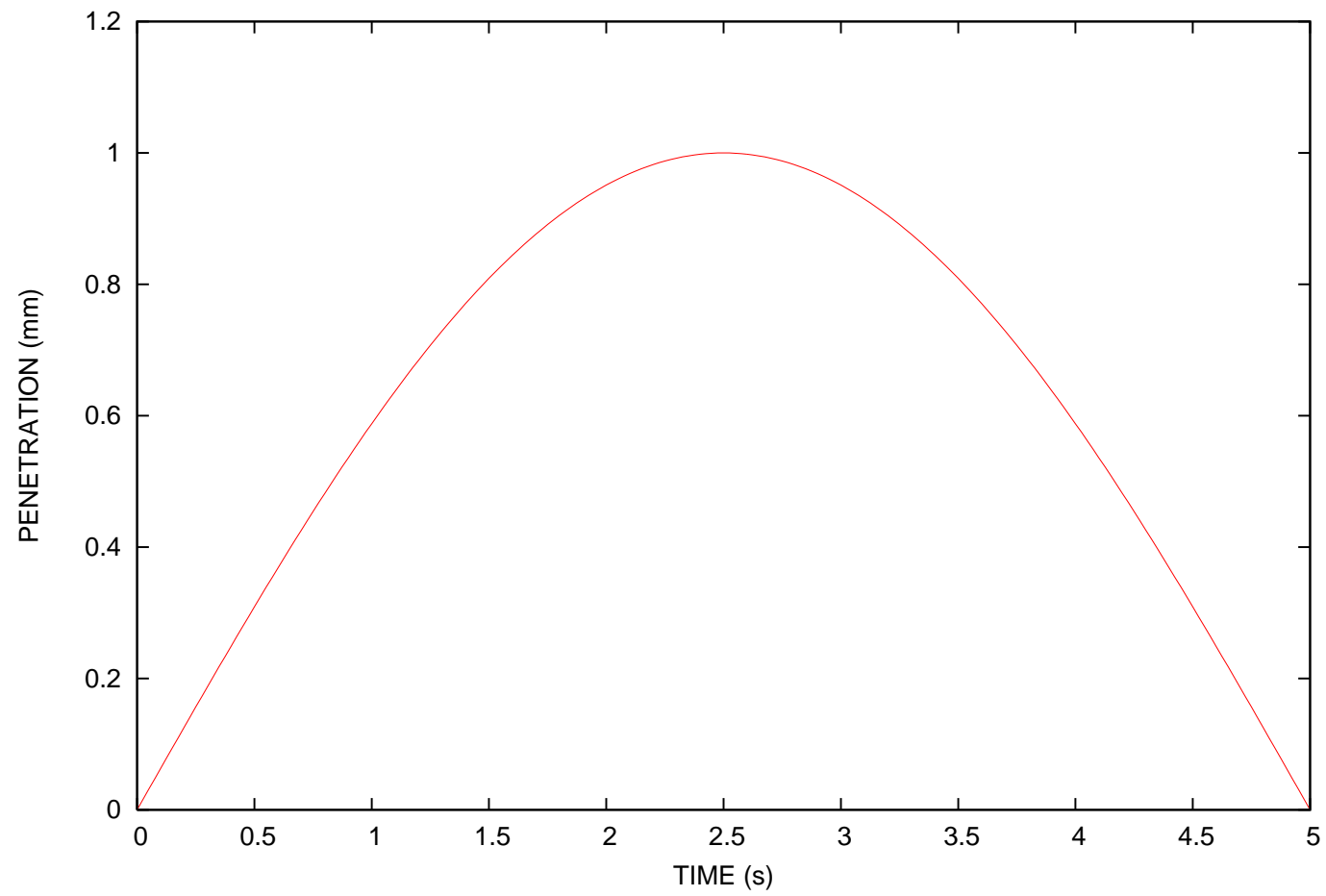

Fig. 5. Spherical case. The penetration $\delta(t)$ is imposed $(a)$ The difference between the contact forces, - central indenter, ------ peripheral indenter $(b)$ The penetration $\delta(t)$ 


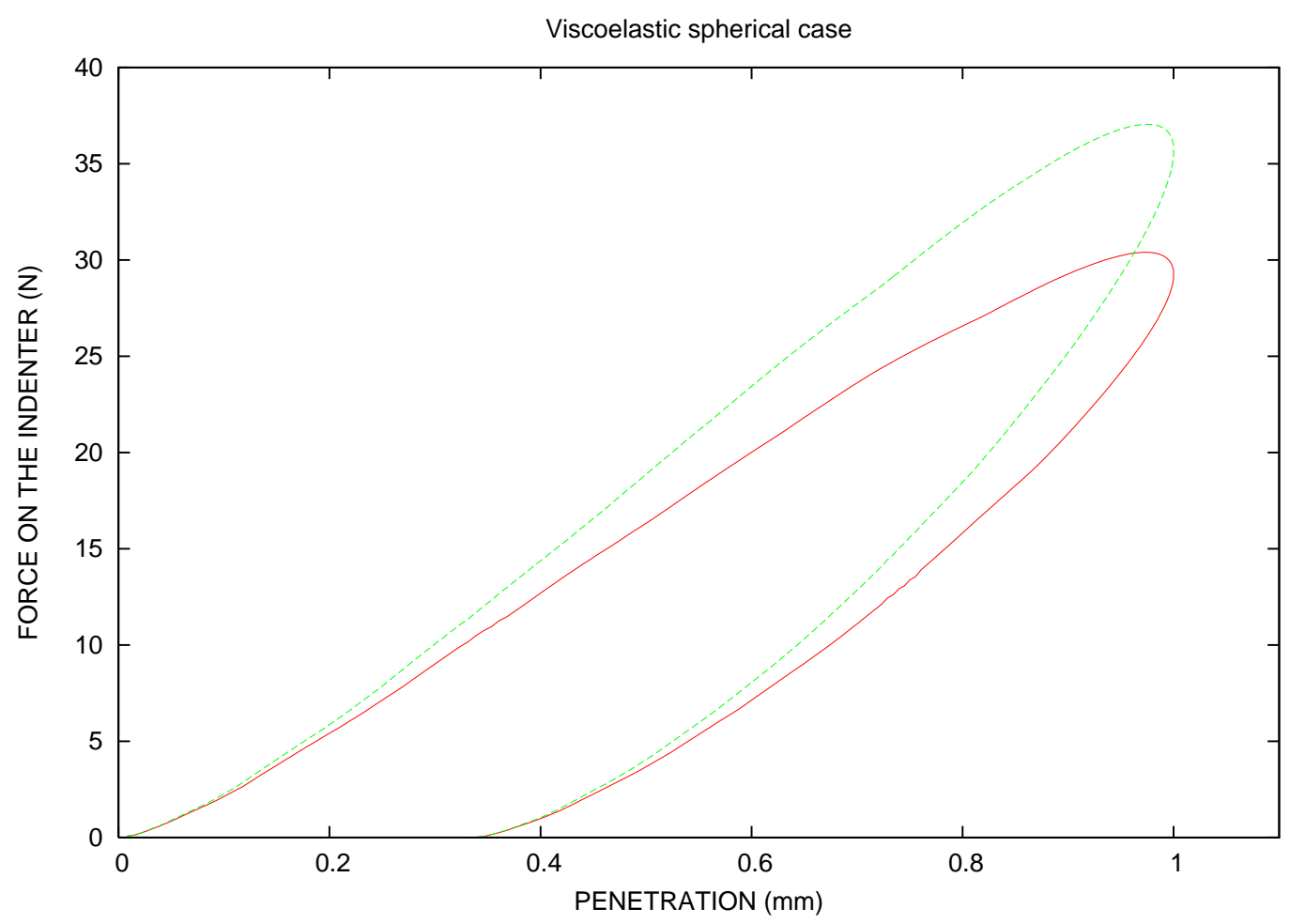

Fig. 6. Spherical case. The difference between the contact forces, — central indenter, ------ peripheral indenter 


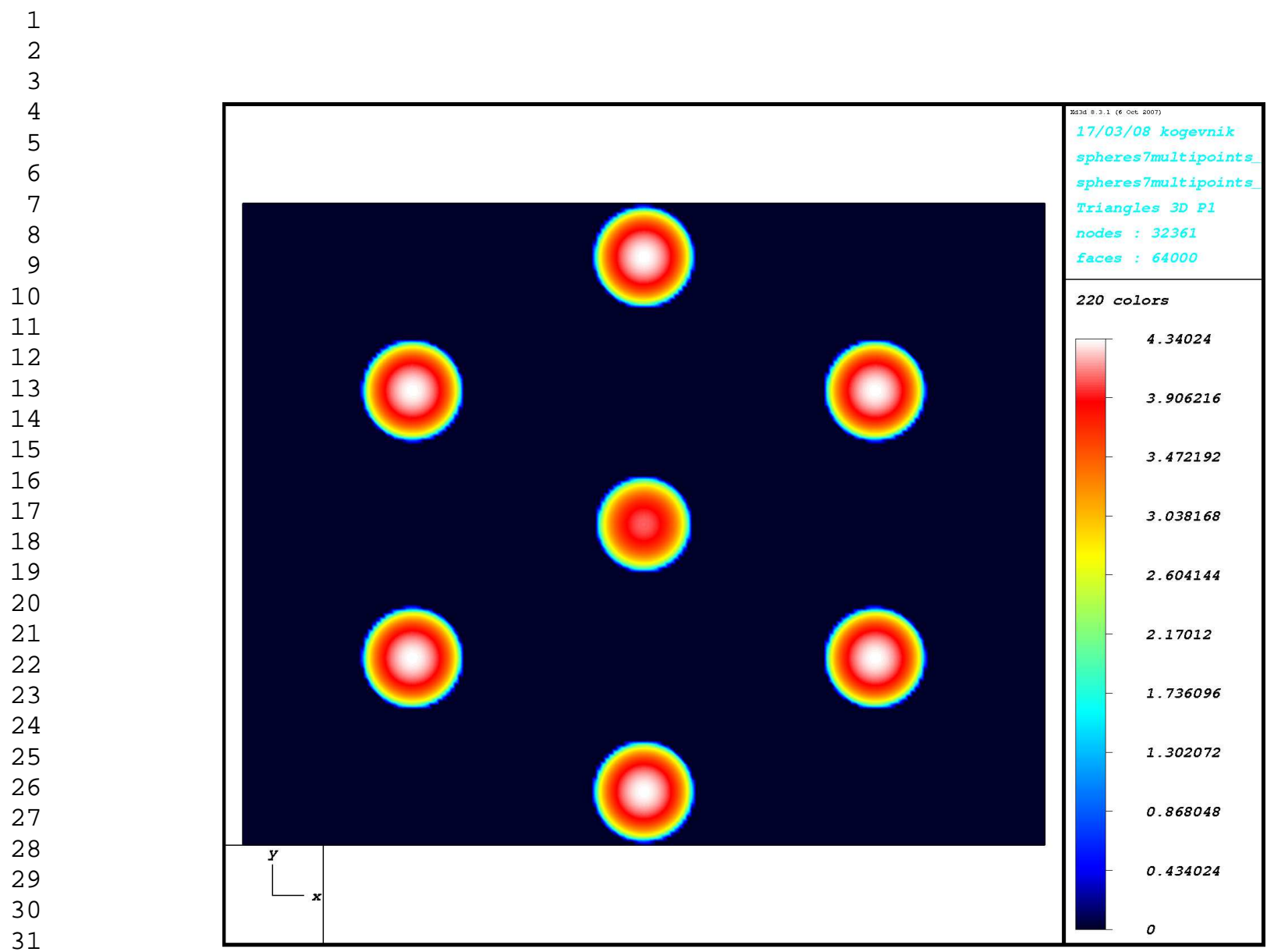




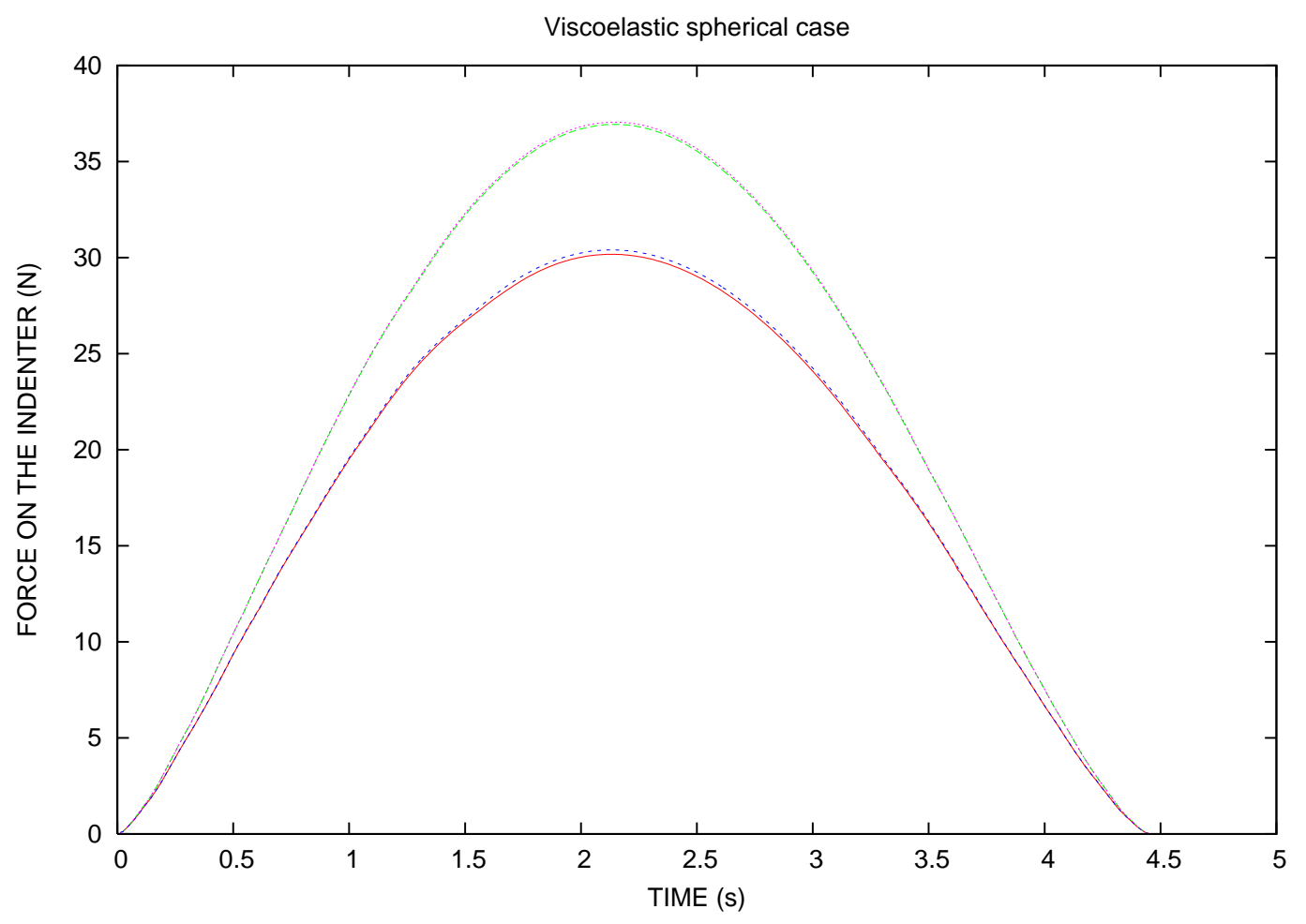

Fig. 8. Spherical case. The penetration $\delta(t)$ is imposed. The difference between the contact forces, — central indenter (Algorithm 1), ------ peripheral indenter (Algorithm 1), …... central indenter (Algorithm 2), ........... peripheral indenter (Algorithm 2) 


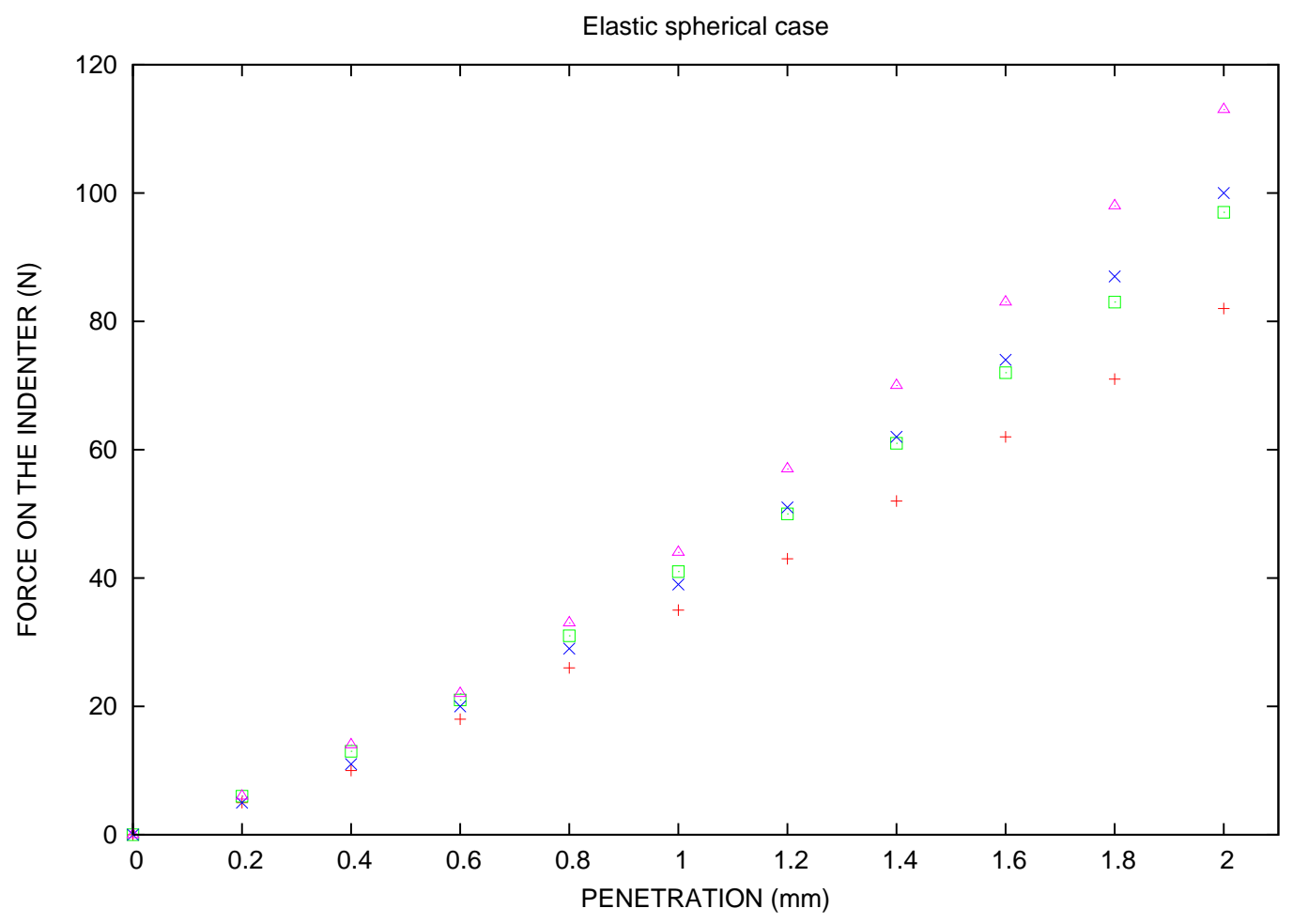

Fig. 9. Spherical case (elastic). The difference between the contact forces, + central indenter $(\mathrm{TIM}), \times$ peripheral indenter (TIM), $\square$ central indenter (FEM), $\triangle$ peripheral indenter (FEM) 

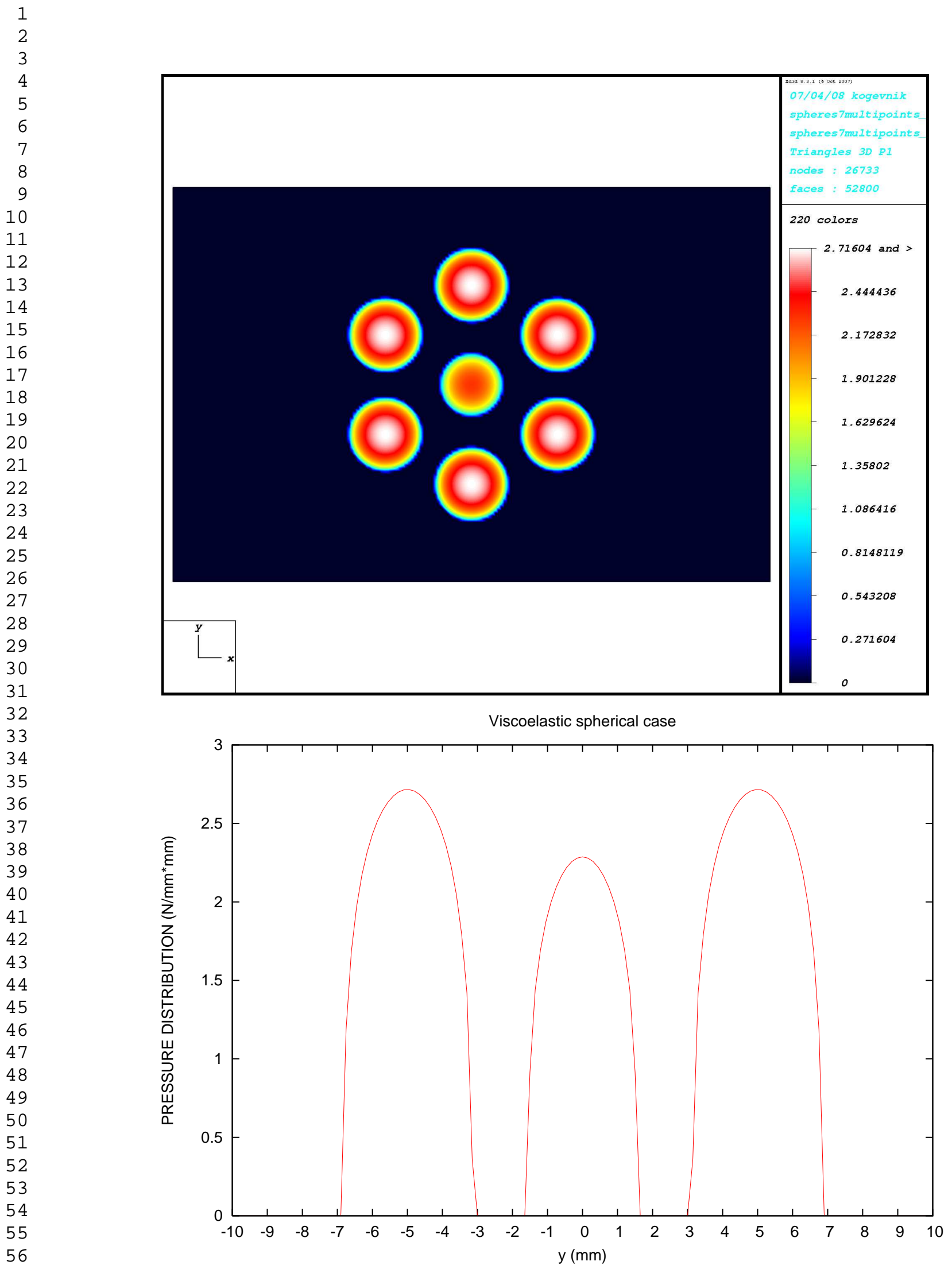

Fig. 10. Spherical-ended cylindrical case. At the top the pressure distribution obtained with Algorithm 2 for $t=1 \mathrm{~s}$ and $a=2.5 \mathrm{~mm}$. At the bottom the pressure for $x=0 \mathrm{~mm}$. 


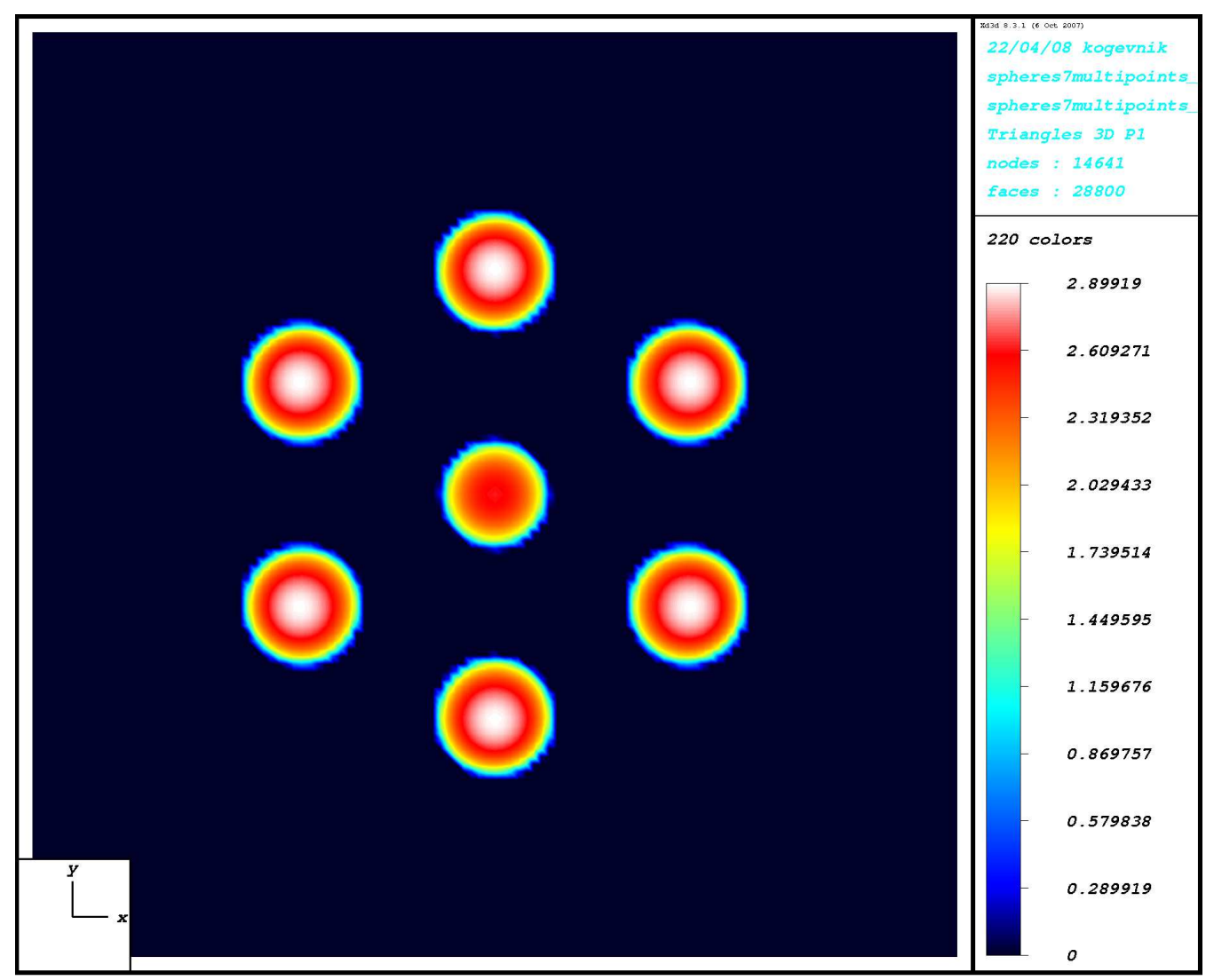

Viscoelastic spherical case

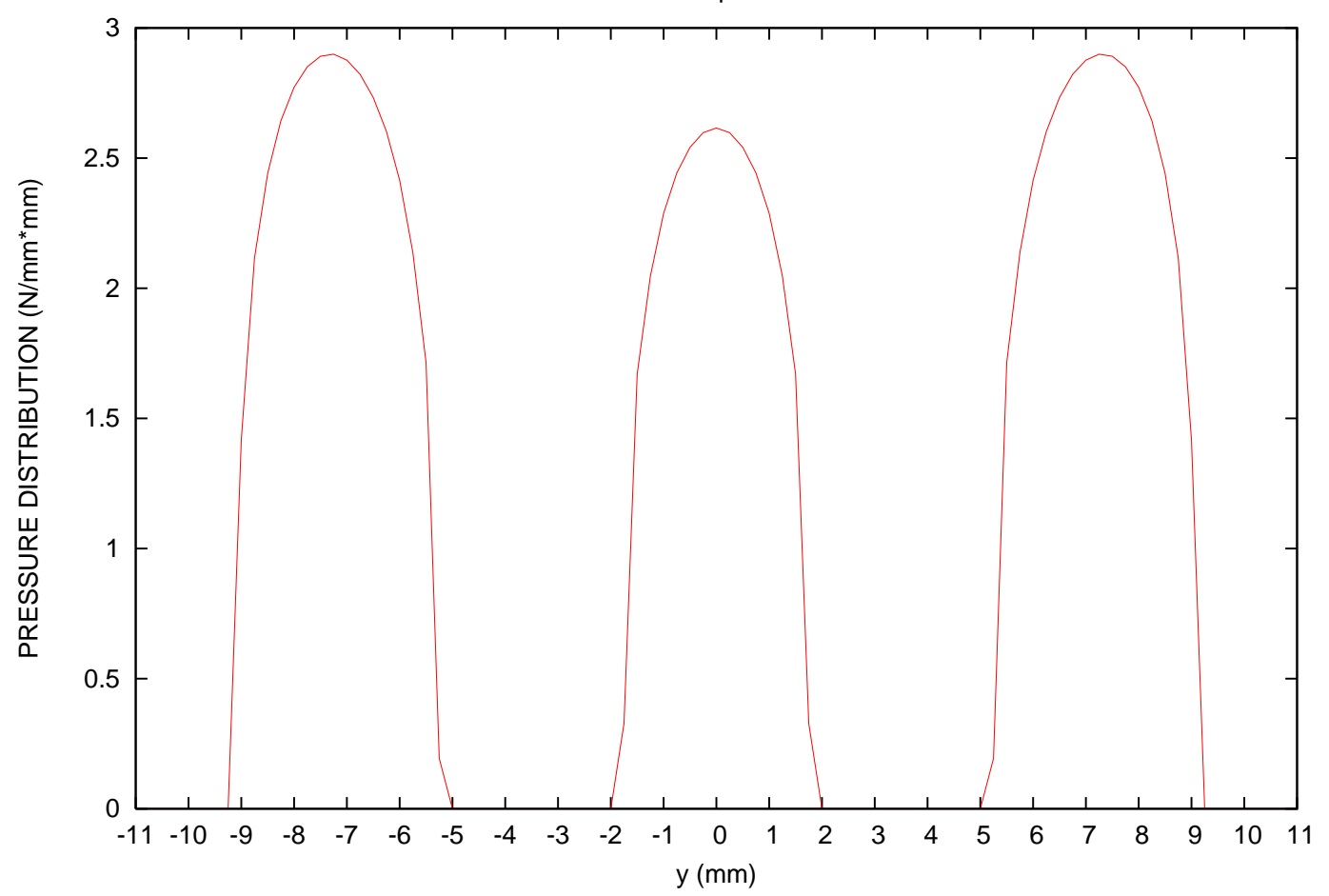

Fig. 11. Spherical-ended cylindrical case. At the top the pressure distribution obtained with Algorithm 1 for $t=1 \mathrm{~s}$ and $a=3.5 \mathrm{~mm}$. At the bottom the pressure for $x=0 \mathrm{~mm}$. 
(a) Viscoelastic spherical case

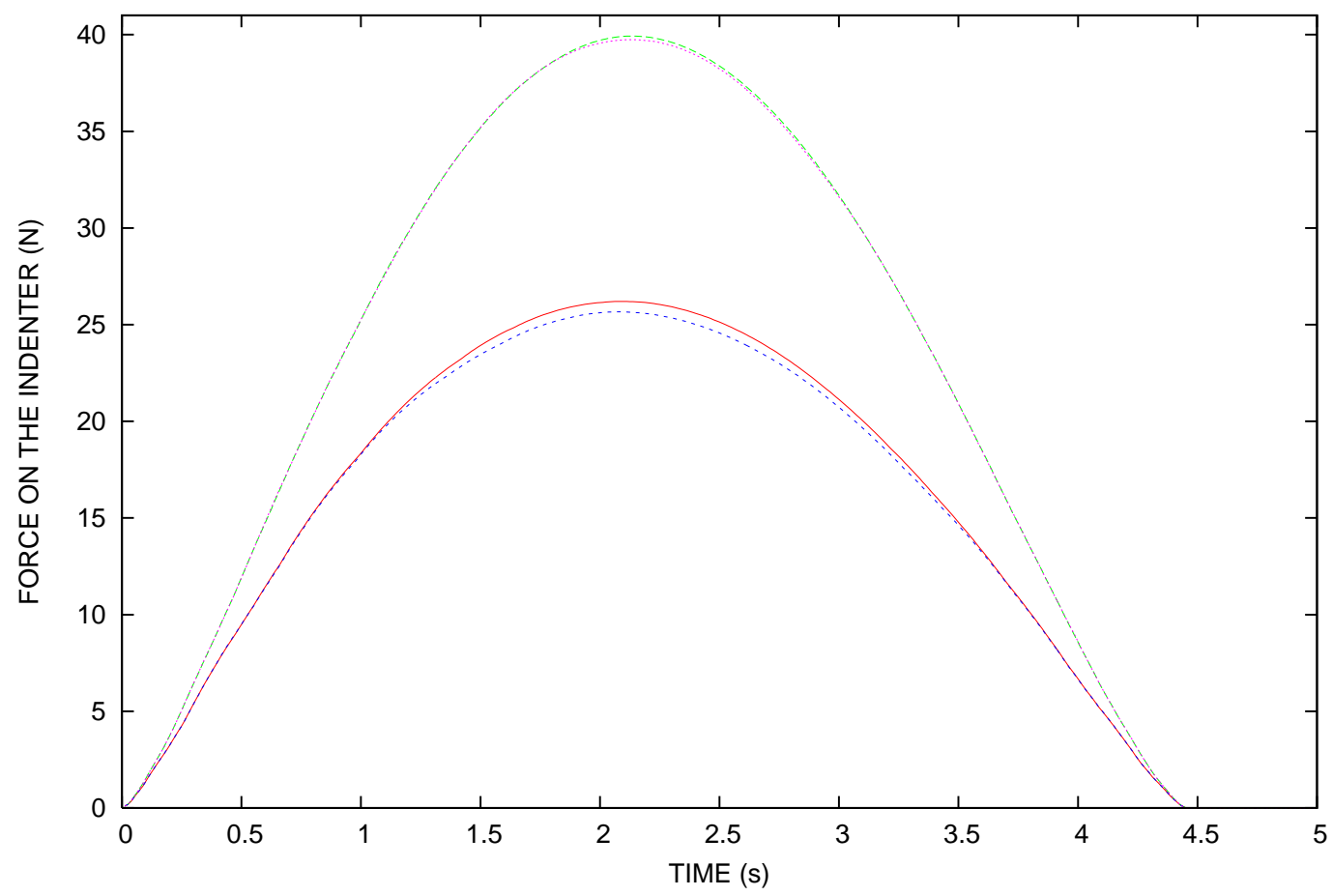

(b) Viscoelastic spherical case

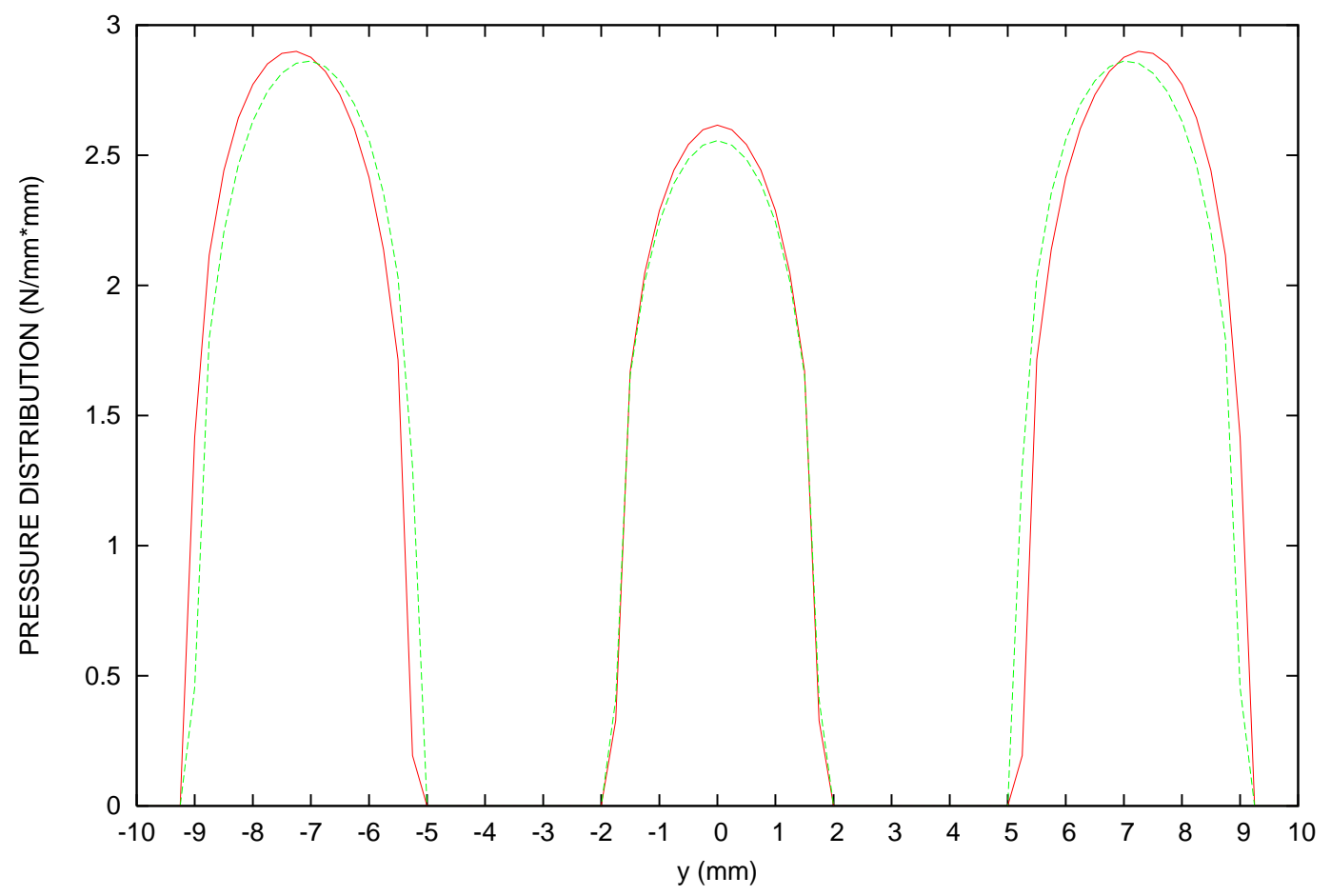

Fig. 12. Spherical-ended cylindrical case. The penetration $\delta(t)$ is imposed. $(a)$ The difference between the contact forces, — central indenter (Algorithm 1), ----peripheral indenter (Algorithm 1), ….. central indenter (Algorithm 2), ........... peripheral indenter (Algorithm 2) (b) The difference between the pressures for $t=1$ $\mathrm{s}, x=0 \mathrm{~mm}$ and $a=3.5 \mathrm{~mm}, \longrightarrow$ Algfifithm 1, ------ Algorithm 2 


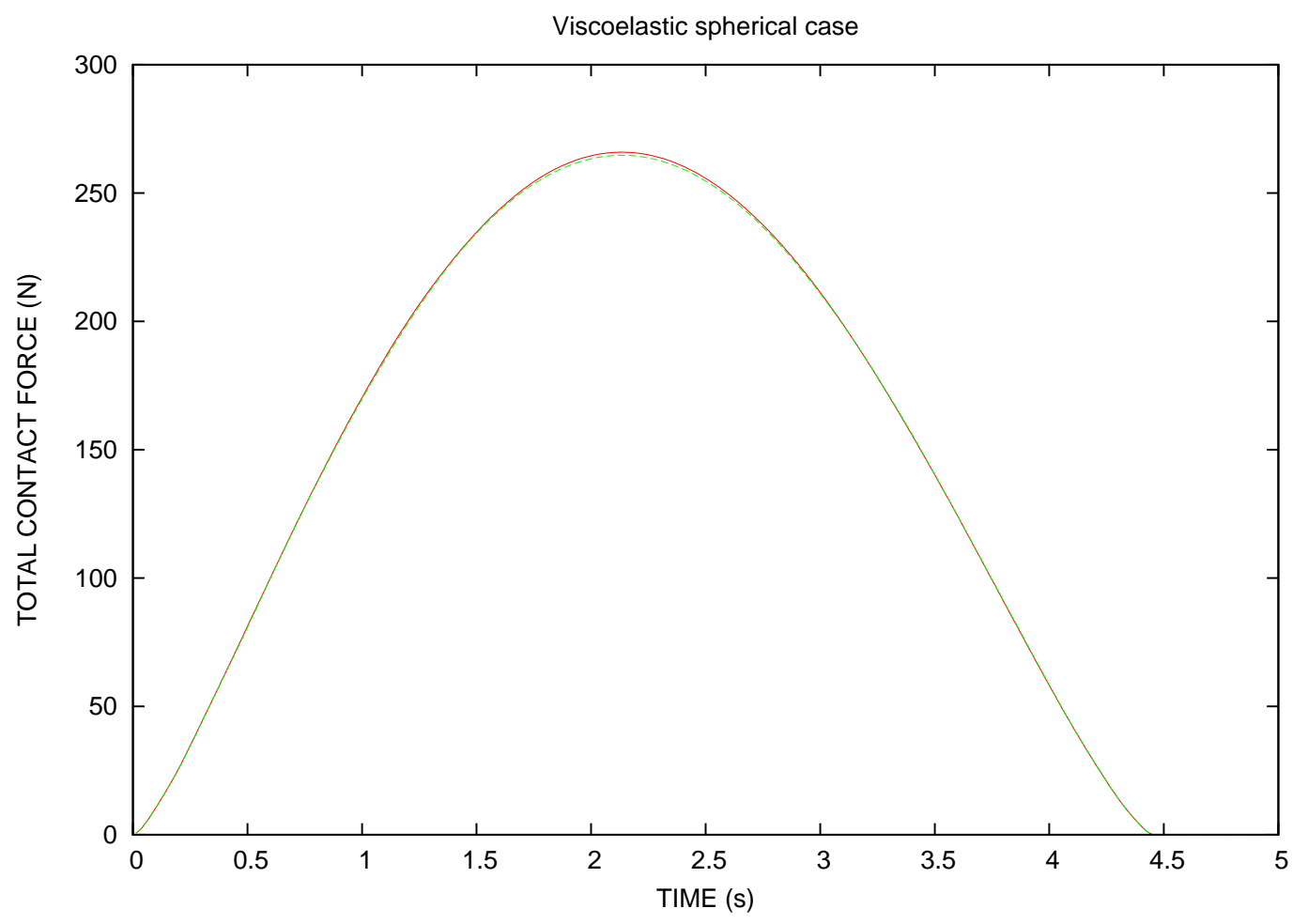

Fig. 13. Spherical-ended cylindrical case. The penetration $\delta(t)$ is imposed. The difference between the contact forces, — MIM, ------ TIM 


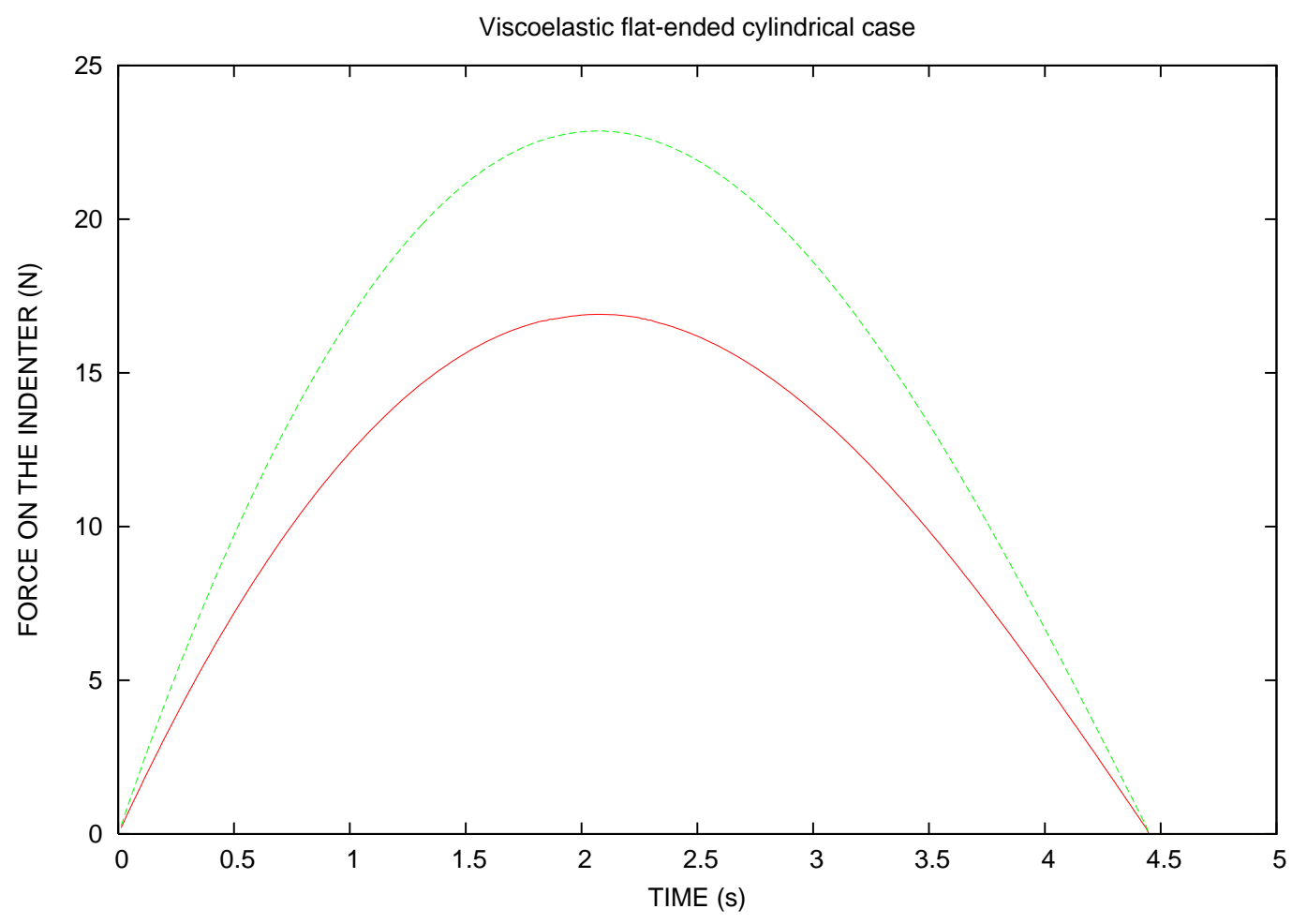

Fig. 14. Flat-ended cylindrical case. The penetration $\delta(t)$ is imposed. The difference between the contact forces, —— central indenter, ------ peripheral indenter 


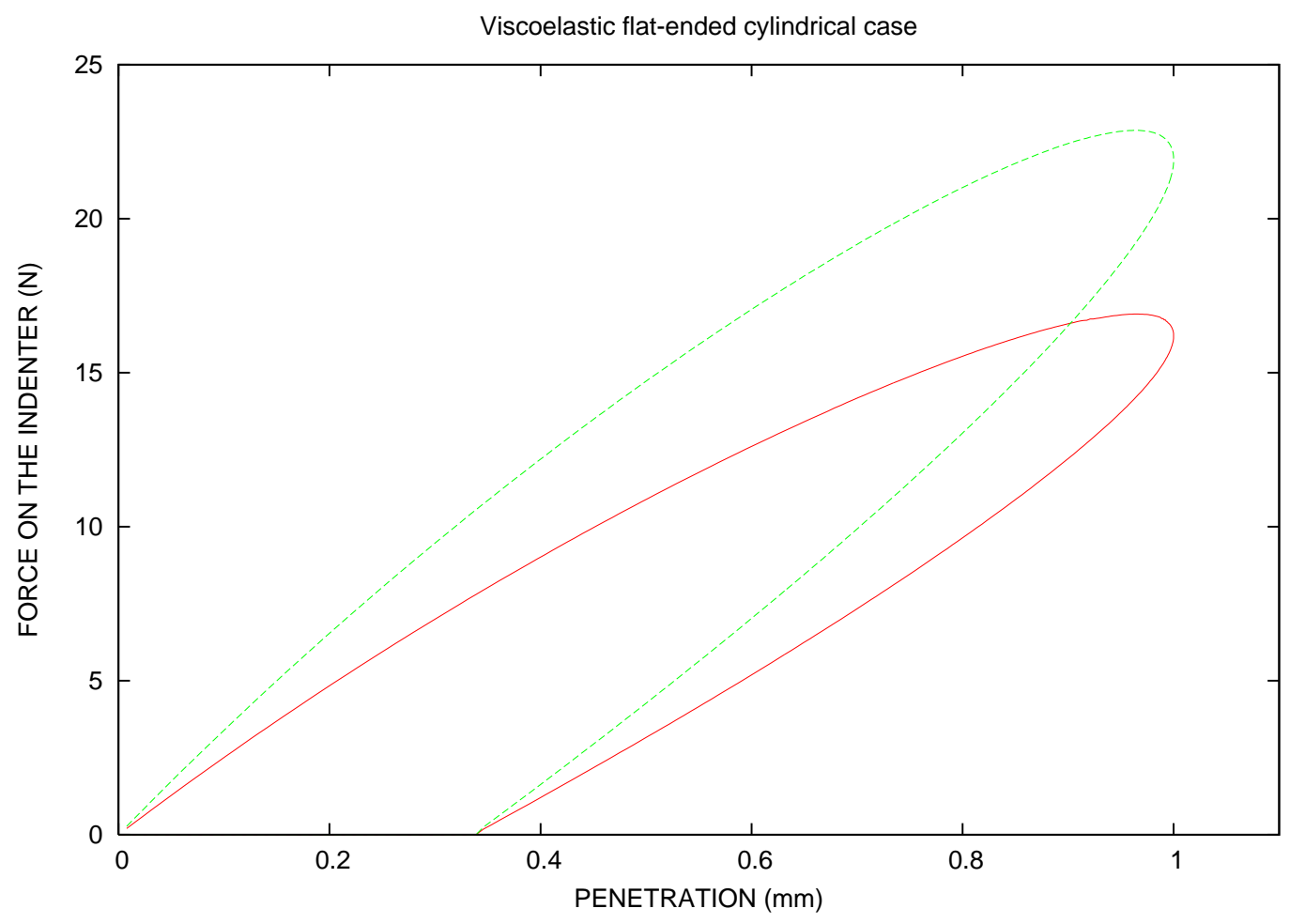

Fig. 15. Flat-ended cylindrical case. The difference between the contact forces, central indenter, ------ peripheral indenter 

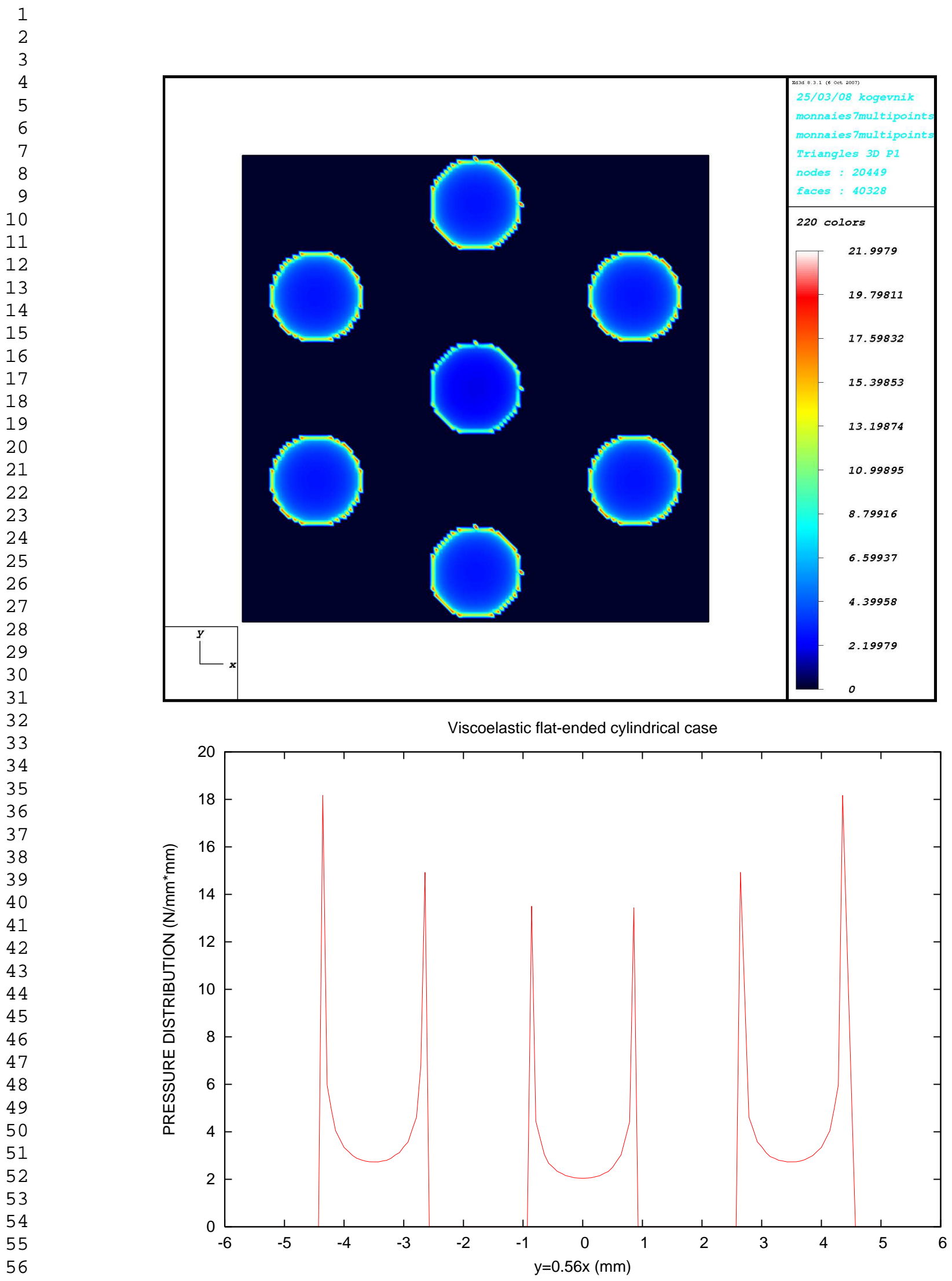

Fig. 16. Flat-ended cylindrical case. At the top the pressure distribution for $t=1$

s. At the bottom the pressure $p(x, 0.56 x, 1)$ 


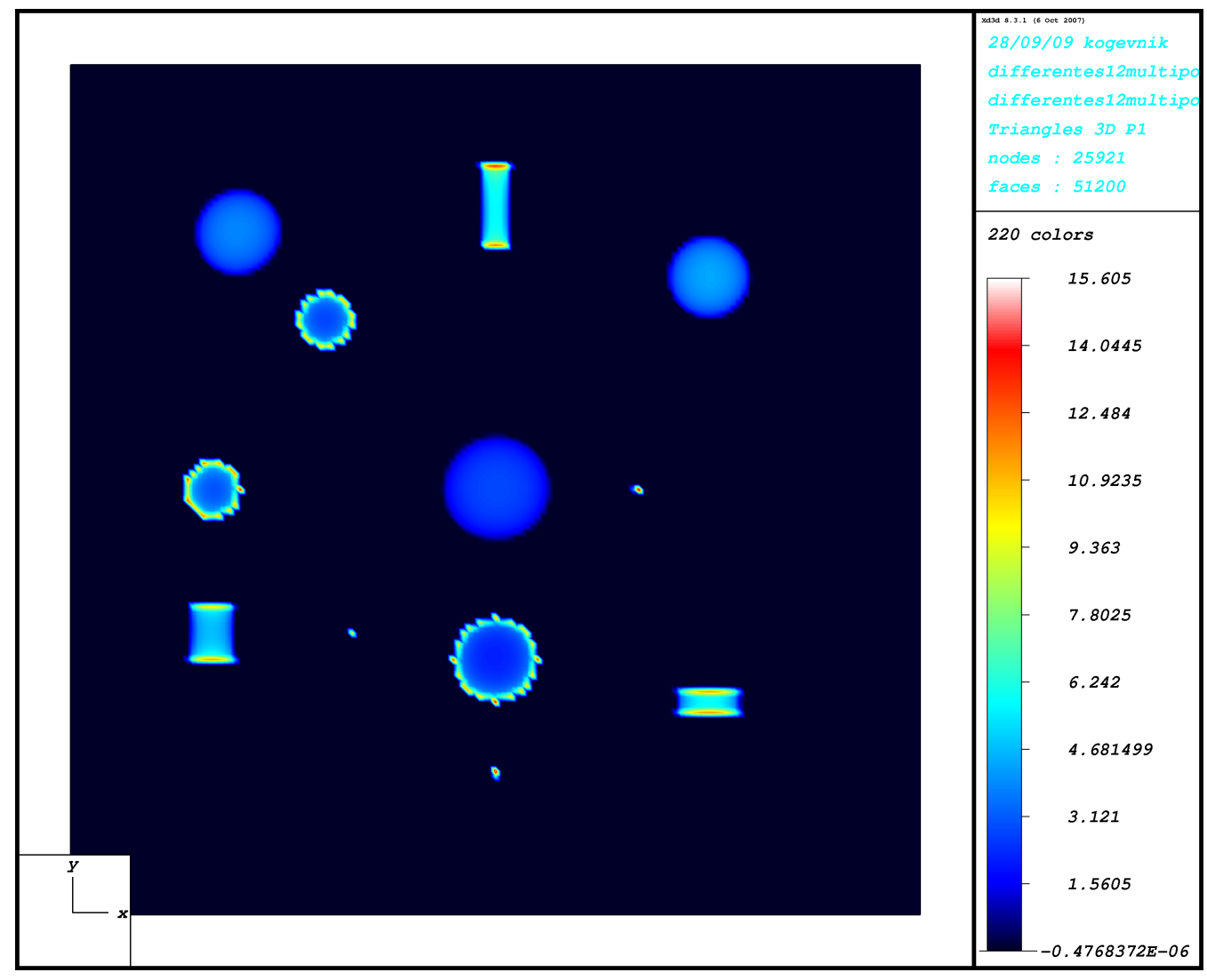

General case

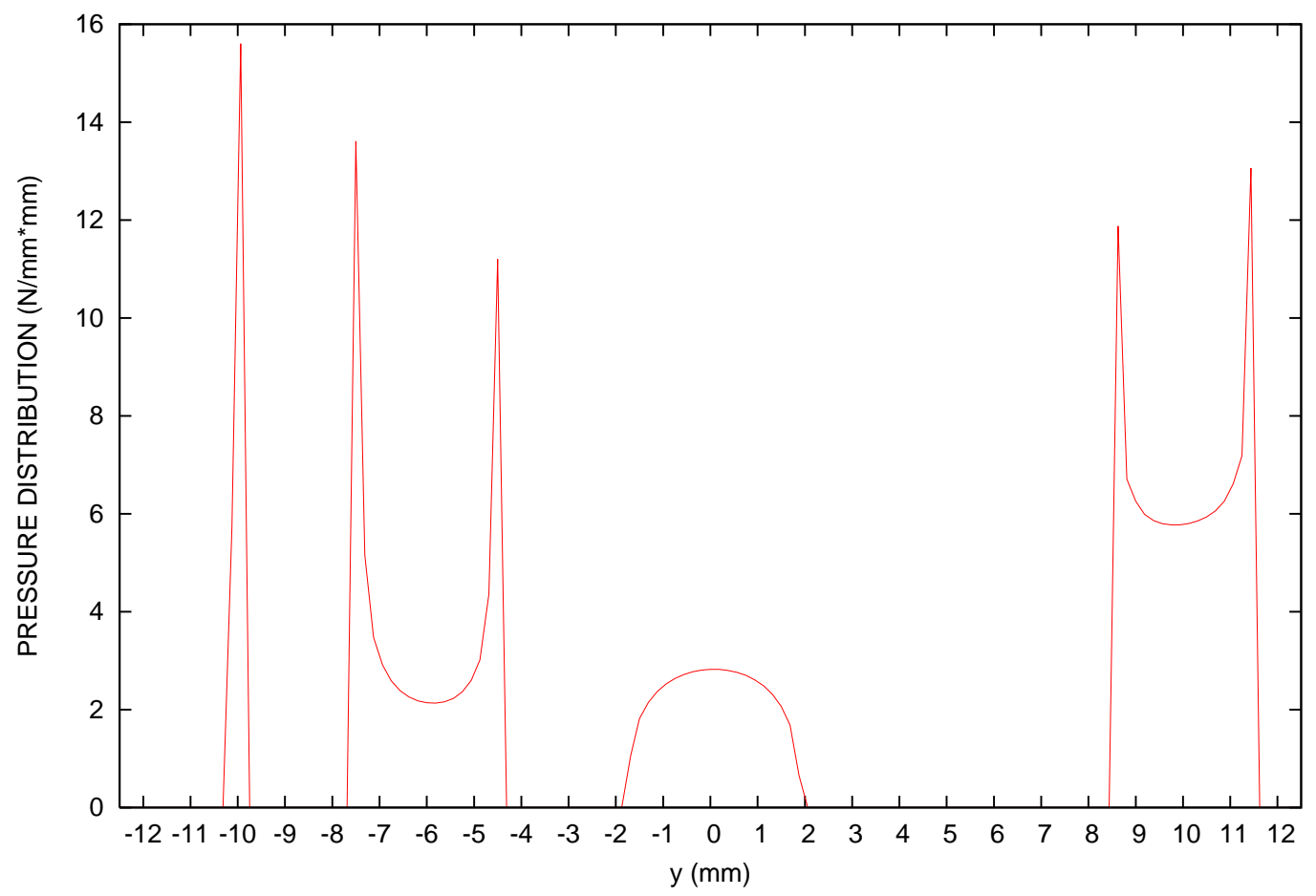

Fig. 17. General case: three spherical-ended cylindrical indenters, three flat-ended cylindrical indenters, three conical indenters, three cylindrical indenters. At the top the pressure distribution for $t=1 \mathrm{~s}$. At the bottom the pressure for $x=0 \mathrm{~mm}$. 